\title{
Reliability Analysis of Random Fuzzy Unrepairable Systems
}

\author{
Ying Liu, ${ }^{1}$ Xiaozhong $\mathrm{Li},{ }^{1}$ and Jianbin $\mathrm{Li}^{2}$ \\ ${ }^{1}$ Department of Computer Sciences, Tianjin University of Science and Technology, Tianjin 300222, China \\ ${ }^{2}$ School of Management, Huazhong University of Science and Technology, Wuhan 430074, China \\ Correspondence should be addressed to Jianbin Li; jbli@hust.edu.cn
}

Received 15 February 2014; Revised 4 April 2014; Accepted 4 April 2014; Published 2 June 2014

Academic Editor: Beatrice Paternoster

Copyright (C) 2014 Ying Liu et al. This is an open access article distributed under the Creative Commons Attribution License, which permits unrestricted use, distribution, and reproduction in any medium, provided the original work is properly cited.

\begin{abstract}
The lifetimes of components in unrepairable systems are considered as random fuzzy variables since randomness and fuzziness are often merged with each other. Then we establish the fundamental mathematical models of random fuzzy unrepairable systems, including series systems, parallel systems, series-parallel systems, parallel-series systems, and cold standby systems with absolutely reliable conversion switches. Furthermore, the expressions of reliability and mean time to failure (MTTF) are given for the above five random fuzzy unrepairable systems, respectively. Finally, numerical examples are given to show the application in a lighting lamp system and a hi-fi system.
\end{abstract}

\section{Introduction}

The conventional reliability theory has been successfully used for solving various reliability problems, in which the lifetimes of systems are assumed to be random variables and the system behavior can be fully characterized by probability theory. It is well-known that reliability and mean time to failure (MTTF) are good evaluations in unrepairable systems, where the reliability is defined by the probability of the random event "system is functioning at time $t$ " and MTTF is the expected value of random lifetime of the system. The results on classical reliability theory can be referred to studies such as Barlow and Proschan [1], Dhillon and Singh [2], Epstein and Sobel [3], Gnedenko et al. [4], Kaufmann [5], Kapur and Lamberson [6], Natarajan [7], Ross [8], Sharma et al. [9], Lin and Yeh [10], Tian et al. [11], Marquez et al. [12], and Hsu et al. [13].

Although the traditional reliability theory has been proved to be effective in many cases, using probability method in engineering problems needs to have three basic premises: firstly, the events should be clearly defined; secondly, there should exist a large number of samples; and thirdly, the samples should have the probability of repetition. If the three premises do not hold, using probability theory to deal with the reliability problems has certain limitations. So fuzzy theory has been introduced to reliability theory by several authors. In 1975, Kaufmann [5] first used fuzzy theory in reliability engineering. Chowdhury and Misra [14] presented a method to find an expression of fuzzy system reliability of a nonseries parallel network taking into consideration the special requirements of fuzzy sets. Cai et al. [15-18] introduced various forms of fuzzy reliability theories, including profust reliability theory, posbist reliability theory, and posfust reliability theory. Their studies can be considered by taking new assumptions, such as the possibility assumption or the fuzzystate assumption, in place of the probability assumption or the binary-state assumption. Utkin $[19,20]$ discussed the fuzzy system reliability based on the binary-state assumption and possibility assumption and considered the fuzzy availability and unavailability and the fuzzy operative availability and unavailability. Utkin and Gurov [21] proposed a general approach on the basis of a system of functional equations according to Cai's theory. In Praba et al. [22], a new method for finding fuzzy system reliability using posfust reliability theory was demonstrated, where the system was modelled as a unified fuzzy Markov model. Cooman [23] introduced the notion of possibilistic structure function based on the concept of the classical two-valued structure function and studied the possibilistic uncertainty of the states of a system and its components. Huang [24] developed the fundamental 
calculation formulas of fuzzy reliability and established the fuzzy reliability models of unrepairable systems. Huang et al. [25] proposed a new method to determine the membership function of the estimates of the parameters and the reliability function of multiparameter lifetime distributions. In Liu et al. [26], reliability and performance assessment for fuzzy multistate elements were considered. Ding and Lisnianski [27] considered a multistate system where performance rates and corresponding state probabilities were presented as fuzzy values. Recently, Jiang and Chen [28] developed a computational model of fuzzy reliability focusing on solving the engineering problems with random general stress and fuzzy general strength. Zhang et al. [29] considered a fuzzy age-dependent replacement policy, in which the lifetimes of components were treated as fuzzy variables. Linda and Manic [30] considered interval type-2 fuzzy voter design for fault tolerant systems.

A more general case in practice is that randomness and fuzziness are merged with each other in one unrepairable system. Many researchers have paid attention to these problems. Wang and Watada [31] considered a renewal reward process with fuzzy random interarrival times and rewards under the $T$-independence associated with any continuous Archimedean $t$-norm. Based on using fuzzy random variables to characterize the lifetimes, Wang and Watada [32] studied the redundancy allocation problems to a fuzzy random parallel-series system. Adduri and Penmetsa [33] made system reliability analysis for mixed uncertain variables which contained both probability distributions and fuzzy membership functions. Utkin and Coolen [34] gave an overview of a lot of methods and models for reliability problems mixed with randomness and fuzziness. Utkin et al. [35] studied a simple one-unit system description in the probability and possibility contexts. According to the situation of randomness and fuzziness existing in the actual project, Li et al. [36] proposed a reliability-credibility model based on fuzzy theory, possibility theory, and credibility theory. Liu et al. [37] considered the fuzzy random reliability of structures based on fuzzy random variables. Random fuzzy theory proposed by Liu [38] mainly uses the average chance measure to evaluate the random fuzzy events. Although many measures proposed by researchers have been used to deal with the behavior of random fuzzy phenomena, they have no self-duality properties. However, a self-duality measure is absolutely needed in both theory and practice. Until today, few people have used random fuzzy theory as the basic mathematical tool to deal with reliability problems. For example, Zhao et al. [39] used random fuzzy theory into renewal process, which results were very useful in repairable system theory. Zhao and Liu [40] provided three types of system performances, in which the lifetimes of redundant systems were treated as random fuzzy variables. Since the important figures of merit for repairable systems were the limited availability, steady state failure frequency, mean time between failures, and mean time to repair, Liu et al. [41] gave the reliability analysis of a random fuzzy repairable series system with independent components. In most cases, the components in the system were dependent. So Liu et al. [42] considered two dependent components, established a random fuzzy shock model and a random fuzzy fatal shock model, and studied the bivariate random fuzzy exponential distribution.

The topic of unrepairable system is an important content in system reliability theory. There are many reasons cannot be repaired, some because of technical reasons, cannot repair; some because of economic reasons, not worth to repair; and some because of making repairable system simplification. In this paper, random fuzzy variables are employed to represent uncertain lifetimes of components in the unrepairable systems. We establish the fundamental mathematical models of random fuzzy unrepairable systems, including series systems, parallel systems, series-parallel systems, parallelseries systems, and cold standby systems with absolutely reliable conversion switches. Furthermore, the expressions of reliability and MTTF are given for the above five systems, respectively. The expressions of reliability and MTTF of the random fuzzy unrepairable systems we arrived at are suitable for stochastic cases and fuzzy cases, which shows that the reliability mathematical models and results in this paper generalize the traditional reliability theory.

The rest of this paper is organized as follows. In Section 2, we recall some basic concepts on fuzzy variables and random fuzzy variables. In Section 3, we establish the fundamental mathematical models of random fuzzy unrepairable systems and give the expressions of reliability and MTTF for each system. Some examples are also presented to illustrate how to calculate the reliability and MTTF of given unrepairable systems, in which the lifetimes of components follow certain probability distributions with fuzzy parameters. In Section 4, the application in a lighting lamp system and a hi-fi system is presented.

\section{Fuzzy Variables and Random Fuzzy Variables}

In this section, we first introduce some basic concepts of fuzzy variables based on the credibility measure.

Definition 1 (B. Liu and Y.-K. Liu [43]). Let $\Theta$ be a nonempty set and let $\mathscr{P}(\Theta)$ be the power set of $\Theta$. The set function is called a credibility measure if it satisfies the following four axioms.

Axiom 1. Consider $\operatorname{Cr}\{\Theta\}=1$.

Axiom 2. Cr is increasing; that is, $\operatorname{Cr}\{A\} \leq \operatorname{Cr}\{B\}$ whenever $A \subset B$.

Axiom 3. Cr is self-dual; that is, $\operatorname{Cr}\{A\}+\operatorname{Cr}\left\{A^{c}\right\}=1$ for any $A \in \mathscr{P}(\Theta)$.

Axiom 4. Consider $\operatorname{Cr}\left\{\cup_{i} A_{i}\right\} \wedge 0.5=\sup _{i} \operatorname{Cr}\left\{A_{i}\right\}$ for any $\left\{A_{i}\right\}$ with $\operatorname{Cr}\left\{A_{i}\right\} \leq 0.5$.

Definition 2 (Liu [44]). A fuzzy variable is defined as a function from the credibility space $(\Theta, \mathscr{P}(\Theta), \mathrm{Cr})$ to the set of real numbers. 
Definition 3 (Liu [44]). Let $\xi$ be a fuzzy variable defined on the credibility space $(\Theta, \mathscr{P}(\Theta), \mathrm{Cr})$. Then its membership function is derived from the credibility measure by

$$
\mu(x)=(2 \operatorname{Cr}\{\xi=x\}) \wedge 1, \quad x \in \Re .
$$

Definition 4 (Liu [44]). A fuzzy variable $\xi$ is said to be positive if $\mathrm{Cr}\{\xi \leq 0\}=0$.

Definition 5 (Liu [45]). Let $\xi$ be a fuzzy variable and $\alpha \in(0,1]$. Then

$$
\xi_{\alpha}^{L}=\inf \{x \mid \mu(x) \geq \alpha\}, \quad \xi_{\alpha}^{U}=\sup \{x \mid \mu(x) \geq \alpha\}
$$

are called the $\alpha$-pessimistic value and the $\alpha$-optimistic value of $\xi$, respectively.

Definition 6 (B. Liu and Y.-K. Liu [43]). Let $\xi$ be a fuzzy variable. The expected value $E[\xi]$ is defined as

$$
E[\xi]=\int_{0}^{+\infty} \operatorname{Cr}\{\xi \geq r\} \mathrm{d} r-\int_{-\infty}^{0} \operatorname{Cr}\{\xi \leq r\} \mathrm{d} r
$$

provided that at least one of the two integrals is finite. In particular, if $\xi$ is a positive fuzzy variable, then $E[\xi]=$ $\int_{0}^{+\infty} \operatorname{Cr}\{\xi \geq r\} \mathrm{d} r$.

Proposition 7 (Y.-K. Liu and B. Liu [46]). Let $\xi$ be a fuzzy variable with finite expected value $E[\xi]$; then one has

$$
E[\xi]=\frac{1}{2} \int_{0}^{1}\left[\xi_{\alpha}^{L}+\xi_{\alpha}^{U}\right] d \alpha
$$

where $\xi_{\alpha}^{L}$ and $\xi_{\alpha}^{U}$ are the $\alpha$-pessimistic value and the $\alpha$ optimistic value of $\xi$, respectively.

Definition 8 (Liu [44]). The fuzzy variables $\xi_{1}, \xi_{2}, \ldots, \xi_{n}$ are said to be independent if

$$
\operatorname{Cr}\left\{\bigcap_{i=1}^{n}\left\{\xi_{i} \in \mathscr{B}_{i}\right\}\right\}=\min _{1 \leq i \leq n} \operatorname{Cr}\left\{\xi_{i} \in \mathscr{B}_{i}\right\}
$$

for any sets $\mathscr{B}_{1}, \mathscr{B}_{2}, \ldots, \mathscr{B}_{n}$ of $\mathfrak{R}$.

Proposition 9 (Y.-K. Liu and B. Liu [46] and Zhao et al. [39]). Let $\xi$ and $\eta$ be two independent fuzzy variables. Then

(i) for any $\alpha \in(0,1],(\xi+\eta)_{\alpha}^{L}=\xi_{\alpha}^{L}+\eta_{\alpha}^{L}$;

(ii) for any $\alpha \in(0,1],(\xi+\eta)_{\alpha}^{U}=\xi_{\alpha}^{U}+\eta_{\alpha}^{U}$.

Furthermore, if $\xi$ and $\eta$ are positive, then

(iii) for any $\alpha \in(0,1],(\xi \cdot \eta)_{\alpha}^{L}=\xi_{\alpha}^{L} \cdot \eta_{\alpha}^{L}$;

(iv) for any $\alpha \in(0,1],(\xi \cdot \eta)_{\alpha}^{U}=\xi_{\alpha}^{U} \cdot \eta_{\alpha}^{U}$.

The concept of the random fuzzy variable was given by Liu [45]. Let $(\Omega, \mathscr{A}, \operatorname{Pr})$ be a probability space and $\mathscr{F}$ a collection of random variables. A random fuzzy variable is defined as a function from a credibility space $(\Theta, \mathscr{P}(\Theta)$, Cr) to a collection of random variables $\mathscr{F}$.
Proposition 10 (Liu [38]). Let $\xi$ be a random fuzzy variable on the credibility space $(\Theta, \mathscr{P}(\Theta), C r)$. Then, for $\theta \in \Theta$, one has

(1) $\operatorname{Pr}\{\xi(\theta) \in \mathscr{B}\}$ is a fuzzy variable for any Borel set $\mathscr{B}$ of $\mathfrak{R}$;

(2) $E[\xi(\theta)]$ is a fuzzy variable provided that $E[\xi(\theta)]$ is finite for any fixed $\theta \in \Theta$.

Example 11. A random fuzzy variable $\xi$ is said to be exponential if for each $\theta, \xi(\theta)$ is an exponentially distributed random variable whose density function is defined as

$$
f_{\xi(\theta)}(t)= \begin{cases}X(\theta) \exp (-X(\theta) t), & \text { if } t \geq 0, \\ 0, & \text { if } t<0,\end{cases}
$$

where $X$ is a positive fuzzy variable defined on the space $\Theta$. An exponentially distributed random fuzzy variable is denoted by $\xi \sim \mathscr{E} \mathscr{X} \mathscr{P}(X)$, and the fuzziness of random fuzzy variable $\xi$ is said to be characterized by fuzzy variable $X$. It follows from Proposition 10 that $\operatorname{Pr}\{\xi(\theta) \geq t\}$ and $E[\xi(\theta)]$ are fuzzy variables. We can arrive at $\operatorname{Pr}\{\xi(\theta) \geq t\}=\exp (-X(\theta) t)$ and $E[\xi(\theta)]=1 / X(\theta)$.

Definition 12 (Y.-K. Liu and B. Liu [46]). Let $\xi$ be a random fuzzy variable defined on the credibility space $(\Theta, \mathscr{P}(\Theta), \mathrm{Cr})$. Then the expected value $E[\xi]$ is defined by

$$
\begin{aligned}
E[\xi]= & \int_{0}^{+\infty} \operatorname{Cr}\{\theta \in \Theta \mid E[\xi(\theta)] \geq r\} \mathrm{d} r \\
& -\int_{-\infty}^{0} \operatorname{Cr}\{\theta \in \Theta \mid E[\xi(\theta)] \leq r\} \mathrm{d} r
\end{aligned}
$$

provided that at least one of the two integrals is finite. In particular, if $\xi$ is a positive fuzzy variable, then $E[\xi]=$ $\int_{0}^{+\infty} \operatorname{Cr}\{\theta \in \Theta \mid E[\xi(\theta)] \geq r\} \mathrm{d} r$.

Definition 13 (Y.-K. Liu and B. Liu [47]). Let $\xi$ be a random fuzzy variable. Then the average chance, denoted by $\mathrm{Ch}$, of random fuzzy event characterized by $\{\xi \in \mathscr{B}\}$ is defined as

$$
\operatorname{Ch}\{\xi \in \mathscr{B}\}=\int_{0}^{1} \operatorname{Cr}\{\theta \in \Theta \mid \operatorname{Pr}\{\xi(\theta) \in \mathscr{B}\} \geq p\} \mathrm{d} p
$$

Remark 14. If $\xi$ degenerates to a random variable, then the average chance degenerates to $\operatorname{Pr}\{\xi \in \mathscr{B}\}$, which is just the probability of random event. If $\xi$ degenerates to a fuzzy variable, then the average chance degenerates to $\operatorname{Cr}\{\xi \in \mathscr{B}\}$, which is just the credibility of fuzzy event.

Finally, we refer to a definition on the stochastic ordering which is usually employed in the comparison of the lifetimes of systems.

Definition 15 (Ross [48]). A collection of random variables $\mathscr{F}$ is said to be a totally ordered set with stochastic ordering if and only if, for any given $\zeta_{1}, \zeta_{2} \in \mathscr{F}$, and $r \in \mathfrak{R}$, either

$$
\operatorname{Pr}\left\{\zeta_{1} \leq r\right\} \leq \operatorname{Pr}\left\{\zeta_{2} \leq r\right\} \quad\left(\text { denoted by } \zeta_{2} \leq_{d} \zeta_{1}\right)
$$


or

$$
\operatorname{Pr}\left\{\zeta_{1} \leq r\right\} \geq \operatorname{Pr}\left\{\zeta_{2} \leq r\right\} \quad\left(\text { denoted by } \zeta_{1} \leq_{d} \zeta_{2}\right)
$$

Remark 16 (Ross [48]). For any given $\zeta_{1}, \zeta_{2} \in \mathscr{F}$, we have

$$
E\left[\zeta_{1}\right] \leq E\left[\zeta_{2}\right] \Longleftrightarrow \zeta_{1} \leq_{d} \zeta_{2}
$$

\section{Random Fuzzy Unrepairable Systems}

In this section, we first define the reliability and MTTF of random fuzzy unrepairable systems. Then the reliability and MTTF of random fuzzy series systems, parallel systems, series-parallel systems, parallel-series systems, and cold standby systems are discussed, respectively.

Definition 17. Let $X$ be the random fuzzy lifetime of an unrepairable system, which is defined on the credibility space $(\Theta, \mathscr{P}(\Theta), \mathrm{Cr})$; then the reliability of the unrepairable system is defined by

$$
R(t)=\operatorname{Ch}\{X \geq t\}
$$

Definition 18. Let $X$ be the random fuzzy lifetime of an unrepairable system, which is defined on the credibility space $(\Theta, \mathscr{P}(\Theta), \mathrm{Cr})$; then MTTF of the unrepairable system is defined by

$$
\operatorname{MTTF}=\int_{0}^{+\infty} \operatorname{Cr}\{\theta \in \Theta \mid E[X(\theta)] \geq r\} \mathrm{d} r .
$$

3.1. Reliability Analysis of Random Fuzzy Unrepairable Series Systems. Consider a series system composed of $n$ independent components. Let $X_{i}$ be the lifetime of component $i$, which is a random fuzzy variable on the credibility space $\left(\Theta_{i}, \mathscr{P}\left(\Theta_{i}\right), \mathrm{Cr}_{i}\right), i=1,2, \ldots, n$. Obviously, the lifetime of the series system is $X=\min \left\{X_{1}, X_{2}, \ldots, X_{n}\right\}$, which is a random fuzzy variable on the product credibility space $(\Theta, \mathscr{P}(\Theta), \mathrm{Cr})$, where $\Theta=\Theta_{1} \times \Theta_{2} \times \cdots \times \Theta_{n}$ and $\mathrm{Cr}=\mathrm{Cr}_{1} \wedge \mathrm{Cr}_{2} \wedge \cdots \wedge \mathrm{Cr}_{n}$.

Theorem 19. Let $X_{i}, i=1,2, \ldots, n$ be random fuzzy variables. Assume that the $\alpha$-pessimistic values and the $\alpha$-optimistic values of $E\left[X_{i}\left(\theta_{i}\right)\right], i=1,2, \ldots, n$, are continuous at the point $\alpha, \alpha \in(0,1]$. The reliability of random fuzzy series system is

$$
R(t)=E\left[\prod_{i=1}^{n} \operatorname{Pr}\left\{\omega \in \Omega \mid X_{i}\left(\theta_{i}\right)(\omega) \geq t\right\}\right]
$$

Proof. By Definitions 13 and 17 and Proposition 7, we have

$$
\begin{aligned}
R(t)= & \operatorname{Ch}\{X \geq t\} \\
= & \int_{0}^{1} \operatorname{Cr}\{\theta \in \Theta \mid \operatorname{Pr}\{X(\theta) \geq t\} \geq p\} \mathrm{d} p \\
= & \frac{1}{2} \int_{0}^{1}\left(\operatorname{Pr}_{\alpha}^{L}\{\omega \in \Omega \mid X(\theta)(\omega) \geq t\}\right. \\
& \left.\quad+\operatorname{Pr}_{\alpha}^{U}\{\omega \in \Omega \mid X(\theta)(\omega) \geq t\}\right) \mathrm{d} \alpha .
\end{aligned}
$$

Let $A_{i}=\left\{\theta_{i} \in \Theta_{i} \mid \mu\left\{\theta_{i}\right\} \geq \alpha\right\}, i=1,2, \ldots, n$. Since the $\alpha$-pessimistic values and the $\alpha$-optimistic values of fuzzy variables $E\left[X_{i}\left(\theta_{i}\right)\right], \theta_{i} \in \Theta_{i}, i=1,2, \ldots, n$, are continuous at the point $\alpha, \alpha \in(0,1]$, then there exist maximum and minimum values; that is, at least there exist points $\theta_{i}^{\prime}, \theta_{i}^{\prime \prime} \in A_{i}$ such that

$$
E\left[X_{i}\left(\theta_{i}^{\prime}\right)\right]=E\left[X_{i}\left(\theta_{i}\right)\right]_{\alpha}^{L}, \quad E\left[X_{i}\left(\theta_{i}^{\prime \prime}\right)\right]=E\left[X_{i}\left(\theta_{i}\right)\right]_{\alpha}^{U}
$$

For any $\theta_{i, \alpha} \in A_{i}$, we have

$$
E\left[X_{i}\left(\theta_{i}^{\prime}\right)\right] \leq E\left[X_{i}\left(\theta_{i, \alpha}\right)\right] \leq E\left[X_{i}\left(\theta_{i}^{\prime \prime}\right)\right], \quad i=1,2, \ldots, n
$$

Hence, by Remark 16, we have

$$
X_{i}\left(\theta_{i}^{\prime}\right) \leq_{d} X_{i}\left(\theta_{i, \alpha}\right) \leq_{d} X_{i}\left(\theta_{i}^{\prime \prime}\right), \quad \theta_{i, \alpha} \in A_{i}, i=1,2, \ldots, n
$$

It follows from Definition 15 that

$$
\begin{aligned}
\operatorname{Pr}\{\omega & \left.\in \Omega \mid X_{i}\left(\theta_{i}^{\prime}\right)(\omega) \geq t\right\} \\
& \leq \operatorname{Pr}\left\{\omega \in \Omega \mid X_{i}\left(\theta_{i, \alpha}\right)(\omega) \geq t\right\} \\
& \leq \operatorname{Pr}\left\{\omega \in \Omega \mid X_{i}\left(\theta_{i}^{\prime \prime}\right)(\omega) \geq t\right\}, \quad i=1,2, \ldots, n .
\end{aligned}
$$

It is easy to see that

$$
\begin{aligned}
\prod_{i=1}^{n} \operatorname{Pr} & \left\{\omega \in \Omega \mid X_{i}\left(\theta_{i}^{\prime}\right)(\omega) \geq t\right\} \\
& \leq \prod_{i=1}^{n} \operatorname{Pr}\left\{\omega \in \Omega \mid X_{i}\left(\theta_{i, \alpha}\right)(\omega) \geq t\right\} \\
& \leq \prod_{i=1}^{n} \operatorname{Pr}\left\{\omega \in \Omega \mid X_{i}\left(\theta_{i}^{\prime \prime}\right)(\omega) \geq t\right\}
\end{aligned}
$$

that is,

$$
\begin{aligned}
& \operatorname{Pr}\left\{\omega \in \Omega \mid \min \left\{X_{1}\left(\theta_{1}^{\prime}\right)(\omega), \ldots, X_{n}\left(\theta_{n}^{\prime}\right)(\omega)\right\} \geq t\right\} \\
& \quad \leq \operatorname{Pr}\left\{\omega \in \Omega \mid \min \left\{X_{1}\left(\theta_{1, \alpha}\right)(\omega), \ldots, X_{n}\left(\theta_{n, \alpha}\right)(\omega)\right\} \geq t\right\} \\
& \quad \leq \operatorname{Pr}\left\{\omega \in \Omega \mid \min \left\{X_{1}\left(\theta_{1}^{\prime \prime}\right)(\omega), \ldots, X_{n}\left(\theta_{n}^{\prime \prime}\right)(\omega)\right\} \geq t\right\} .
\end{aligned}
$$


Since $\theta_{i, \alpha}$ are arbitrary points in $A_{i}, i=1,2, \ldots, n$, by (21), we have

$$
\begin{aligned}
& \operatorname{Pr}_{\alpha}^{L}\{\omega \in \Omega \mid X(\theta)(\omega) \geq t\} \\
& \quad=\operatorname{Pr}\left\{\omega \in \Omega \mid \min \left\{X_{1}\left(\theta_{1}^{\prime}\right)(\omega), \ldots, X_{n}\left(\theta_{n}^{\prime}\right)(\omega)\right\} \geq t\right\} \\
& \quad=\prod_{i=1}^{n} \operatorname{Pr}\left\{\omega \in \Omega \mid X_{i}\left(\theta_{i}^{\prime}\right)(\omega) \geq t\right\}, \\
& \operatorname{Pr}_{\alpha}^{U}\{\omega \in \Omega \mid X(\theta)(\omega) \geq t\} \\
& \quad=\operatorname{Pr}\left\{\omega \in \Omega \mid \min \left\{X_{1}\left(\theta_{1}^{\prime \prime}\right)(\omega), \ldots, X_{n}\left(\theta_{n}^{\prime \prime}\right)(\omega)\right\} \geq t\right\} \\
& \quad=\prod_{i=1}^{n} \operatorname{Pr}\left\{\omega \in \Omega \mid X_{i}\left(\theta_{i}^{\prime \prime}\right)(\omega) \geq t\right\} .
\end{aligned}
$$

On the other hand, by (19), we have

$$
\begin{aligned}
& \operatorname{Pr}_{\alpha}^{L}\{\omega\left.\in \Omega \mid X_{i}\left(\theta_{i}\right)(\omega) \geq t\right\}=\operatorname{Pr}\left\{\omega \in \Omega \mid X_{i}\left(\theta_{i}^{\prime}\right)(\omega) \geq t\right\}, \\
& \operatorname{Pr}_{\alpha}^{U}\left\{\omega \in \Omega \mid X_{i}\left(\theta_{i}\right)(\omega) \geq t\right\} \\
& \quad=\operatorname{Pr}\left\{\omega \in \Omega \mid X_{i}\left(\theta_{i}^{\prime \prime}\right)(\omega) \geq t\right\}, \quad i=1,2, \ldots, n .
\end{aligned}
$$

By (22) and (23), we have

$$
\begin{aligned}
\operatorname{Pr}_{\alpha}^{L}\{\omega & \in \Omega \mid X(\theta)(\omega) \geq t\} \\
& =\prod_{i=1}^{n} \operatorname{Pr}_{\alpha}^{L}\left\{\omega \in \Omega \mid X_{i}\left(\theta_{i}\right)(\omega) \geq t\right\}, \\
\operatorname{Pr}_{\alpha}^{U}\{\omega & \in \Omega \mid X(\theta)(\omega) \geq t\} \\
& =\prod_{i=1}^{n} \operatorname{Pr}_{\alpha}^{U}\left\{\omega \in \Omega \mid X_{i}\left(\theta_{i}\right)(\omega) \geq t\right\} .
\end{aligned}
$$

By (15) and (24), we have

$$
\begin{aligned}
R(t)=\frac{1}{2} \int_{0}^{1}\left(\prod_{i=1}^{n} \operatorname{Pr}_{\alpha}^{L}\left\{\omega \in \Omega \mid X_{i}\left(\theta_{i}\right)(\omega) \geq t\right\}\right. \\
\left.+\prod_{i=1}^{n} \operatorname{Pr}_{\alpha}^{U}\left\{\omega \in \Omega \mid X_{i}\left(\theta_{i}\right)(\omega) \geq t\right\}\right) \mathrm{d} \alpha \\
=\frac{1}{2} \int_{0}^{1}\left(\left[\prod_{i=1}^{n} \operatorname{Pr}\left\{\omega \in \Omega \mid X_{i}\left(\theta_{i}\right)(\omega) \geq t\right\}\right]_{\alpha}^{L}\right. \\
\left.\quad+\left[\prod_{i=1}^{n} \operatorname{Pr}\left\{\omega \in \Omega \mid X_{i}\left(\theta_{i}\right)(\omega) \geq t\right\}\right]_{\alpha}^{U}\right) \mathrm{d} \alpha \\
=E\left[\prod_{i=1}^{n} \operatorname{Pr}\left\{\omega \in \Omega \mid X_{i}\left(\theta_{i}\right)(\omega) \geq t\right\}\right]
\end{aligned}
$$

which completes the proof.
Remark 20. If $X_{i}, i=1,2, \ldots, n$, degenerate to random variables, the result in Theorem 19 degenerates to the form

$$
\begin{aligned}
& R(t) \\
& \quad=\operatorname{Pr}\left\{\omega \in \Omega \mid \min \left\{X_{1}\left(\omega_{1}\right), X_{2}\left(\omega_{2}\right), \ldots, X_{n}\left(\omega_{n}\right)\right\} \geq t\right\} \\
& \quad=\prod_{i=1}^{n} \operatorname{Pr}\left\{X_{i} \geq t\right\},
\end{aligned}
$$

which is consistent with the result in stochastic case (see Barlow and Proschan [1]).

Remark 21. If $X_{i}, i=1,2, \ldots, n$, degenerate to fuzzy variables, the result in Theorem 19 degenerates to the form

$$
\begin{aligned}
R(t) & =\operatorname{Cr}\left\{\theta \in \Theta \mid \min \left\{X_{1}\left(\theta_{1}\right), X_{2}\left(\theta_{2}\right), \ldots, X_{n}\left(\theta_{n}\right)\right\} \geq t\right\} \\
& =\min _{1 \leq i \leq n} \operatorname{Cr}\left\{X_{i}>t\right\}
\end{aligned}
$$

which is consistent with the result in fuzzy case (see Liu and Zhu [49]).

Theorem 22. Let $X_{i}, i=1,2, \ldots, n$, be random fuzzy variables. Assume that the $\alpha$-pessimistic values and the $\alpha$ optimistic values of $E\left[X_{i}\left(\theta_{i}\right)\right], i=1,2, \ldots, n$, are continuous at the point $\alpha, \alpha \in(0,1]$. The MTTF of the series system is

$$
\operatorname{MTTF}=\int_{0}^{+\infty} R(t) d t
$$

Proof. By Definition 18 and Proposition 7, we have

$$
\begin{aligned}
\mathrm{MTTF} & =\int_{0}^{+\infty} \operatorname{Cr}\{\theta \in \Theta \mid E[X(\theta)] \geq r\} \mathrm{d} r \\
& =\frac{1}{2} \int_{0}^{1}\left(E[X(\theta)]_{\alpha}^{L}+E[X(\theta)]_{\alpha}^{U}\right) \mathrm{d} \alpha .
\end{aligned}
$$

By (21), we know that

$$
\begin{aligned}
\min & \left\{X_{1}\left(\theta_{1}^{\prime}\right)(\omega), \ldots, X_{n}\left(\theta_{n}^{\prime}\right)(\omega)\right\} \\
& \leq_{d} \min \left\{X_{1}\left(\theta_{1, \alpha}\right)(\omega), \ldots, X_{n}\left(\theta_{n, \alpha}\right)(\omega)\right\} \\
& \leq_{d} \min \left\{X_{1}\left(\theta_{1}^{\prime \prime}\right)(\omega), \ldots, X_{n}\left(\theta_{n}^{\prime \prime}\right)(\omega)\right\} .
\end{aligned}
$$

It follows from Remark 16 that

$$
\begin{aligned}
E[\min & \left.\left\{X_{1}\left(\theta_{1}^{\prime}\right)(\omega), \ldots, X_{n}\left(\theta_{n}^{\prime}\right)(\omega)\right\}\right] \\
\leq & E\left[\min \left\{X_{1}\left(\theta_{1, \alpha}\right)(\omega), \ldots, X_{n}\left(\theta_{n, \alpha}\right)(\omega)\right\}\right] \\
\leq & E\left[\min \left\{X_{1}\left(\theta_{1}^{\prime \prime}\right)(\omega), \ldots, X_{n}\left(\theta_{n}^{\prime \prime}\right)(\omega)\right\}\right] .
\end{aligned}
$$


Since $\theta_{i, \alpha}$ are arbitrary points in $A_{i}, i=1,2, \ldots, n$, we have

$$
\begin{aligned}
& E[X(\theta)]_{\alpha}^{L} \\
& \quad=E\left[\min \left\{X_{1}\left(\theta_{1}^{\prime}\right)(\omega), \ldots, X_{n}\left(\theta_{n}^{\prime}\right)(\omega)\right\}\right] \\
& \quad=\int_{0}^{+\infty} \prod_{i=1}^{n} \operatorname{Pr}\left\{\omega \in \Omega \mid X_{i}\left(\theta_{i}^{\prime}\right)(\omega)>t\right\} \mathrm{d} t, \\
& E[X(\theta)]_{\alpha}^{U} \\
& \quad=E\left[\min \left\{X_{1}\left(\theta_{1}^{\prime \prime}\right)(\omega), \ldots, X_{n}\left(\theta_{n}^{\prime \prime}\right)(\omega)\right\}\right] \\
& \quad=\int_{0}^{+\infty} \prod_{i=1}^{n} \operatorname{Pr}\left\{\omega \in \Omega \mid X_{i}\left(\theta_{i}^{\prime \prime}\right)(\omega)>t\right\} \mathrm{d} t .
\end{aligned}
$$

It follows from (23), (29), and (32) that

\section{MTTF}

$$
\begin{aligned}
& =\frac{1}{2} \int_{0}^{1}\left\{\int_{0}^{+\infty} \prod_{i=1}^{n} \operatorname{Pr}\left\{\omega \in \Omega \mid X_{i}\left(\theta_{i}^{\prime}\right)(\omega)>t\right\} \mathrm{d} t\right. \\
& \left.\quad+\int_{0}^{+\infty} \prod_{i=1}^{n} \operatorname{Pr}\left\{\omega \in \Omega \mid X_{i}\left(\theta_{i}^{\prime \prime}\right)(\omega)>t\right\} \mathrm{d} t\right\} \mathrm{d} \alpha \\
& =\frac{1}{2} \int_{0}^{1} \int_{0}^{+\infty}\left\{\prod_{i=1}^{n} \operatorname{Pr}_{\alpha}^{L}\left\{\omega \in \Omega \mid X_{i}\left(\theta_{i}\right)(\omega) \geq t\right\}\right. \\
& \left.\quad+\prod_{i=1}^{n} \operatorname{Pr}_{\alpha}^{U}\left\{\omega \in \Omega \mid X_{i}\left(\theta_{i}\right)(\omega) \geq t\right\}\right\} \mathrm{d} t \mathrm{~d} \alpha \\
& =\int_{0}^{+\infty} \frac{1}{2} \int_{0}^{1}\left\{\prod_{i=1}^{n} \operatorname{Pr}_{\alpha}^{L}\left\{\omega \in \Omega \mid X_{i}\left(\theta_{i}\right)(\omega) \geq t\right\}\right. \\
& \left.+\prod_{i=1}^{n} \operatorname{Pr}_{\alpha}^{U}\left\{\omega \in \Omega \mid X_{i}\left(\theta_{i}\right)(\omega) \geq t\right\}\right\} \mathrm{d} \alpha \mathrm{d} t \\
& =\int_{0}^{+\infty} R(t) \mathrm{d} t .
\end{aligned}
$$

The theorem is proved.

Remark 23. If $X_{i}, i=1,2, \ldots, n$, degenerate to random variables, the result in Theorem 22 degenerates to the form

$$
\operatorname{MTTF}=\int_{0}^{+\infty} R(t) \mathrm{d} t
$$

in which $R(t)$ is the reliability of series system in stochastic case.

Remark 24. If $X_{i}, i=1,2, \ldots, n$, degenerate to fuzzy variables, the result in Theorem 22 degenerates to the form

$$
\operatorname{MTTF}=\int_{0}^{+\infty} R(t) \mathrm{d} t
$$

in which $R(t)$ is the reliability of series system in fuzzy case.
Example 25. If the random fuzzy variable $X_{i} \sim \mathscr{E} \mathscr{X} \mathscr{P}\left(\lambda_{i}\right)$, where $\lambda_{i}$ is a fuzzy variable on $\left(\Theta_{i}, \mathscr{P}\left(\Theta_{i}\right), \mathrm{Cr}_{i}\right), i=1,2, \ldots, n$, then we can arrive at

$$
\begin{aligned}
& \operatorname{Pr}_{\alpha}^{L}\{\omega \in \Omega \mid X(\theta)(\omega) \geq t\}=\prod_{i=1}^{n} e^{-\lambda_{i, \alpha}^{U} t}=e^{-\sum_{i=1}^{n} \lambda_{i, \alpha}^{U} t}, \\
& \operatorname{Pr}_{\alpha}^{U}\{\omega \in \Omega \mid X(\theta)(\omega) \geq t\}=\prod_{i=1}^{n} e^{-\lambda_{i, \alpha}^{L} t}=e^{-\sum_{i=1}^{n} \lambda_{i, \alpha}^{L} t} .
\end{aligned}
$$

By Theorems 19 and 22, we have

$$
\begin{gathered}
R(t)=e^{-\sum_{i=1}^{n} E \lambda_{i} t}, \\
\text { MTTF }=\frac{1}{\sum_{i=1}^{n} E \lambda_{i}},
\end{gathered}
$$

in which " $E$ " is the expected value operator of fuzzy variable.

3.2. Reliability Analysis of Random Fuzzy Unrepairable Parallel Systems. Consider a parallel system composed of $n$ independent components. Let $X_{i}$ be the lifetime of component $i$, which is a random fuzzy variable on the credibility space $\left(\Theta_{i}, \mathscr{P}\left(\Theta_{i}\right), \mathrm{Cr}_{i}\right), i=1,2, \ldots, n$. Obviously, the lifetime of the parallel system is $X=\max \left\{X_{1}, X_{2}, \ldots, X_{n}\right\}$, which is a random fuzzy variable on the product credibility space $(\Theta, \mathscr{P}(\Theta), \mathrm{Cr})$, where $\Theta=\Theta_{1} \times \Theta_{2} \times \cdots \times \Theta_{n}$ and $\mathrm{Cr}=$ $\mathrm{Cr}_{1} \wedge \mathrm{Cr}_{2} \wedge \cdots \wedge \mathrm{Cr}_{n}$.

Theorem 26. Let $X_{i}, i=1,2, \ldots, n$, be random fuzzy variables. Assume that the $\alpha$-pessimistic values and the $\alpha$ optimistic values of $E\left[X_{i}\left(\theta_{i}\right)\right], i=1,2, \ldots, n$, are continuous at the point $\alpha, \alpha \in(0,1]$. The reliability of the random fuzzy parallel system is

$$
R(t)=1-E\left\{\prod_{i=1}^{n}\left[1-\operatorname{Pr}\left\{\omega \in \Omega \mid X_{i}\left(\theta_{i}\right)(\omega) \geq t\right\}\right]\right\} .
$$

Proof. By Definitions 13 and 17 and Proposition 7, we have

$$
\begin{aligned}
R(t)= & \operatorname{Ch}\{X \geq t\} \\
= & \int_{0}^{1} \operatorname{Cr}\{\theta \in \Theta \mid \operatorname{Pr}\{X(\theta) \geq t\} \geq p\} \mathrm{d} p \\
= & \frac{1}{2} \int_{0}^{1}\left(\operatorname{Pr}_{\alpha}^{L}\{\omega \in \Omega \mid X(\theta)(\omega) \geq t\}\right. \\
& \left.\quad+\operatorname{Pr}_{\alpha}^{U}\{\omega \in \Omega \mid X(\theta)(\omega) \geq t\}\right) \mathrm{d} \alpha .
\end{aligned}
$$

Let $A_{i}=\left\{\theta_{i} \in \Theta_{i} \mid \mu\left\{\theta_{i}\right\} \geq \alpha\right\}, i=1,2, \ldots, n$. Since the $\alpha$-pessimistic values and the $\alpha$-optimistic values of fuzzy variables $E\left[X_{i}\left(\theta_{i}\right)\right], \theta_{i} \in \Theta_{i}, i=1,2, \ldots, n$, are continuous at the point $\alpha, \alpha \in(0,1]$, then there exist maximum and minimum values; that is, at least there exist points $\theta_{i}^{\prime}, \theta_{i}^{\prime \prime} \in A_{i}$ such that

$$
E\left[X_{i}\left(\theta_{i}^{\prime}\right)\right]=E\left[X_{i}\left(\theta_{i}\right)\right]_{\alpha}^{L}, \quad E\left[X_{i}\left(\theta_{i}^{\prime \prime}\right)\right]=E\left[X_{i}\left(\theta_{i}\right)\right]_{\alpha}^{U} .
$$


For any $\theta_{i, \alpha} \in A_{i}$, we have

$$
E\left[X_{i}\left(\theta_{i}^{\prime}\right)\right] \leq E\left[X_{i}\left(\theta_{i, \alpha}\right)\right] \leq E\left[X_{i}\left(\theta_{i}^{\prime \prime}\right)\right], \quad i=1,2, \ldots, n .
$$

Hence, by Remark 16 we have

$$
X_{i}\left(\theta_{i}^{\prime}\right) \leq_{d} X_{i}\left(\theta_{i, \alpha}\right) \leq_{d} X_{i}\left(\theta_{i}^{\prime \prime}\right), \quad \theta_{i, \alpha} \in A_{i}, i=1,2, \ldots, n .
$$

It follows from Definition 15 that

$$
\begin{aligned}
\operatorname{Pr} & \left\{\omega \in \Omega \mid X_{i}\left(\theta_{i}^{\prime}\right)(\omega) \geq t\right\} \\
& \leq \operatorname{Pr}\left\{\omega \in \Omega \mid X_{i}\left(\theta_{i, \alpha}\right)(\omega) \geq t\right\} \\
& \leq \operatorname{Pr}\left\{\omega \in \Omega \mid X_{i}\left(\theta_{i}^{\prime \prime}\right)(\omega) \geq t\right\}, \quad i=1,2, \ldots, n .
\end{aligned}
$$

It is easy to see that

$$
\begin{aligned}
1- & \prod_{i=1}^{n}\left[1-\operatorname{Pr}\left\{\omega \in \Omega \mid X_{i}\left(\theta_{i}^{\prime}\right)(\omega)>t\right\}\right] \\
& \leq 1-\prod_{i=1}^{n}\left[1-\operatorname{Pr}\left\{\omega \in \Omega \mid X_{i}\left(\theta_{i, \alpha}\right)(\omega)>t\right\}\right] \\
& \leq 1-\prod_{i=1}^{n}\left[1-\operatorname{Pr}\left\{\omega \in \Omega \mid X_{i}\left(\theta_{i}^{\prime \prime}\right)(\omega)>t\right\}\right] ;
\end{aligned}
$$

that is,

$$
\begin{aligned}
\operatorname{Pr} & \left\{\omega \in \Omega \mid \max \left\{X_{1}\left(\theta_{1}^{\prime}\right)(\omega), \ldots, X_{n}\left(\theta_{n}^{\prime}\right)(\omega)\right\}>t\right\} \\
& \leq \operatorname{Pr}\left\{\omega \in \Omega \mid \max \left\{X_{1}\left(\theta_{1, \alpha}\right)(\omega), \ldots, X_{n}\left(\theta_{n, \alpha}\right)(\omega)\right\}>t\right\} \\
& \leq \operatorname{Pr}\left\{\omega \in \Omega \mid \max \left\{X_{1}\left(\theta_{1}^{\prime \prime}\right)(\omega), \ldots, X_{n}\left(\theta_{n}^{\prime \prime}\right)(\omega)\right\}>t\right\} .
\end{aligned}
$$

Since $\theta_{i, \alpha}$ are arbitrary points in $A_{i}, i=1,2, \ldots, n$, by (45), we have

$$
\begin{aligned}
& \operatorname{Pr}_{\alpha}^{L}\{\omega \in \Omega \mid X(\theta)(\omega) \geq t\} \\
& \quad=\operatorname{Pr}\left\{\omega \in \Omega \mid \max \left\{X_{1}\left(\theta_{1}^{\prime}\right)(\omega), \ldots, X_{n}\left(\theta_{n}^{\prime}\right)(\omega)\right\}>t\right\} \\
& \quad=1-\prod_{i=1}^{n}\left[1-\operatorname{Pr}\left\{\omega \in \Omega \mid X_{i}\left(\theta_{i}^{\prime}\right)(\omega)>t\right\}\right], \\
& \operatorname{Pr}_{\alpha}^{U}\{\omega \in \Omega \mid X(\theta)(\omega) \geq t\} \\
& \quad=\operatorname{Pr}\left\{\omega \in \Omega \mid \max \left\{X_{1}\left(\theta_{1}^{\prime \prime}\right)(\omega), \ldots, X_{n}\left(\theta_{n}^{\prime \prime}\right)(\omega)\right\}>t\right\} \\
& \quad=1-\prod_{i=1}^{n}\left[1-\operatorname{Pr}\left\{\omega \in \Omega \mid X_{i}\left(\theta_{i}^{\prime \prime}\right)(\omega)>t\right\}\right] .
\end{aligned}
$$

On the other hand, by (19), we have

$$
\begin{aligned}
& \operatorname{Pr}_{\alpha}^{L}\{\omega\left.\in \Omega \mid X_{i}\left(\theta_{i}\right)(\omega) \geq t\right\}=\operatorname{Pr}\left\{\omega \in \Omega \mid X_{i}\left(\theta_{i}^{\prime}\right)(\omega)>t\right\}, \\
& \operatorname{Pr}_{\alpha}^{U}\left\{\omega \in \Omega \mid X_{i}\left(\theta_{i}\right)(\omega) \geq t\right\} \\
& \quad=\operatorname{Pr}\left\{\omega \in \Omega \mid X_{i}\left(\theta_{i}^{\prime \prime}\right)(\omega)>t\right\}, \quad i=1,2, \ldots, n .
\end{aligned}
$$

By (46) and (47), we have

$$
\operatorname{Pr}_{\alpha}^{L}\{\omega \in \Omega \mid X(\theta)(\omega) \geq t\}
$$

$$
=1-\prod_{i=1}^{n}\left[1-\operatorname{Pr}_{\alpha}^{L}\left\{\omega \in \Omega \mid X_{i}\left(\theta_{i}\right)(\omega) \geq t\right\}\right],
$$

$\operatorname{Pr}_{\alpha}^{U}\{\omega \in \Omega \mid X(\theta)(\omega) \geq t\}$

$$
=1-\prod_{i=1}^{n}\left[1-\operatorname{Pr}_{\alpha}^{U}\left\{\omega \in \Omega \mid X_{i}\left(\theta_{i}\right)(\omega) \geq t\right\}\right] .
$$

By (39) and (48) we have

$$
\begin{aligned}
& R(t) \\
& =\frac{1}{2} \int_{0}^{1}\left(1-\prod_{i=1}^{n}\left[1-\operatorname{Pr}_{\alpha}^{L}\left\{\omega \in \Omega \mid X_{i}\left(\theta_{i}\right)(\omega) \geq t\right\}\right]+1\right. \\
& \left.-\prod_{i=1}^{n}\left[1-\operatorname{Pr}_{\alpha}^{U}\left\{\omega \in \Omega \mid X_{i}\left(\theta_{i}\right)(\omega) \geq t\right\}\right]\right) \mathrm{d} \alpha \\
& =1-\frac{1}{2} \\
& \times \int_{0}^{1}\left(\left\{\prod_{i=1}^{n}\left[1-\operatorname{Pr}\left\{\omega \in \Omega \mid X_{i}\left(\theta_{i}\right)(\omega) \geq t\right\}\right]\right\}_{\alpha}^{U}\right. \\
& \left.+\left\{\prod_{i=1}^{n}\left[1-\operatorname{Pr}\left\{\omega \in \Omega \mid X_{i}\left(\theta_{i}\right)(\omega) \geq t\right\}\right]\right\}_{\alpha}^{L}\right) \mathrm{d} \alpha \\
& =1-E\left\{\prod_{i=1}^{n}\left[1-\operatorname{Pr}\left\{\omega \in \Omega \mid X_{i}\left(\theta_{i}\right)(\omega) \geq t\right\}\right]\right\} \text {, }
\end{aligned}
$$

which completes the proof.

Remark 27. If $X_{i}, i=1,2, \ldots, n$, degenerate to random variables, the result in Theorem 26 degenerates to the form

$$
\begin{aligned}
& R(t) \\
& =\operatorname{Pr}\left\{\omega \in \Omega \mid \max \left\{X_{1}\left(\omega_{1}\right), X_{2}\left(\omega_{2}\right), \ldots, X_{n}\left(\omega_{n}\right)\right\} \geq t\right\} \\
& =1-\prod_{i=1}^{n}\left[1-\operatorname{Pr}\left\{X_{i} \geq t\right\}\right],
\end{aligned}
$$

which is consistent with the result in stochastic case (see Barlow and Proschan [1]).

Remark 28. If $X_{i}, i=1,2, \ldots, n$, degenerate to fuzzy variables, the result in Theorem 26 degenerates to the form

$$
\begin{aligned}
R(t) & =\operatorname{Cr}\left\{\theta \in \Theta \mid \max \left\{X_{1}\left(\theta_{1}\right), X_{2}\left(\theta_{2}\right), \ldots, X_{n}\left(\theta_{n}\right)\right\} \geq t\right\} \\
& =\max _{1 \leq i \leq n} \operatorname{Cr}\left\{X_{i}>t\right\},
\end{aligned}
$$

which is consistent with the result in fuzzy case (see Liu and Zhu [49]). 
Theorem 29. Let $X_{i}, i=1,2, \ldots, n$, be random fuzzy variables. Assume that the $\alpha$-pessimistic values and the $\alpha$ optimistic values of $E\left[X_{i}\left(\theta_{i}\right)\right], i=1,2, \ldots, n$, are continuous at the point $\alpha, \alpha \in(0,1]$. The MTTF of the parallel system is

$$
\operatorname{MTTF}=\int_{0}^{+\infty} R(t) d t
$$

Proof. By Definition 18 and Proposition 7, we can see

$$
\begin{aligned}
\text { MTTF } & =\int_{0}^{+\infty} \operatorname{Cr}\{\theta \in \Theta \mid E[X(\theta)] \geq r\} \mathrm{d} r \\
& =\frac{1}{2} \int_{0}^{1}\left(E[X(\theta)]_{\alpha}^{L}+E[X(\theta)]_{\alpha}^{U}\right) \mathrm{d} \alpha .
\end{aligned}
$$

By (45), we know that

$$
\begin{aligned}
\max & \left\{X_{1}\left(\theta_{1}^{\prime}\right)(\omega), \ldots, X_{n}\left(\theta_{n}^{\prime}\right)(\omega)\right\} \\
& \leq_{d} \max \left\{X_{1}\left(\theta_{1, \alpha}\right)(\omega), \ldots, X_{n}\left(\theta_{n, \alpha}\right)(\omega)\right\} \\
& \leq_{d} \max \left\{X_{1}\left(\theta_{1}^{\prime \prime}\right)(\omega), \ldots, X_{n}\left(\theta_{n}^{\prime \prime}\right)(\omega)\right\} .
\end{aligned}
$$

It follows from Remark 16 that

$$
\begin{aligned}
E[\max & \left.\left\{X_{1}\left(\theta_{1}^{\prime}\right)(\omega), \ldots, X_{n}\left(\theta_{n}^{\prime}\right)(\omega)\right\}\right] \\
\leq & E\left[\max \left\{X_{1}\left(\theta_{1, \alpha}\right)(\omega), \ldots, X_{n}\left(\theta_{n, \alpha}\right)(\omega)\right\}\right] \\
\leq & E\left[\max \left\{X_{1}\left(\theta_{1}^{\prime \prime}\right)(\omega), \ldots, X_{n}\left(\theta_{n}^{\prime \prime}\right)(\omega)\right\}\right] .
\end{aligned}
$$

Since $\theta_{i, \alpha}$ are arbitrary points in $A_{i}, i=1,2, \ldots, n$, we have

$$
\begin{aligned}
& E[X(\theta)]_{\alpha}^{L} \\
& =E\left[\max \left\{X_{1}\left(\theta_{1}^{\prime}\right)(\omega), \ldots, X_{n}\left(\theta_{n}^{\prime}\right)(\omega)\right\}\right] \\
& =\int_{0}^{+\infty}\left\{1-\prod_{i=1}^{n}\left[1-\operatorname{Pr}\left\{\omega \in \Omega \mid X_{i}\left(\theta_{i}^{\prime}\right)(\omega)>t\right\}\right]\right\} \mathrm{d} t, \\
& E[X(\theta)]_{\alpha}^{U} \\
& \quad=E\left[\max \left\{X_{1}\left(\theta_{1}^{\prime \prime}\right)(\omega), \ldots, X_{n}\left(\theta_{n}^{\prime \prime}\right)(\omega)\right\}\right] \\
& \quad=\int_{0}^{+\infty}\left\{1-\prod_{i=1}^{n}\left[1-\operatorname{Pr}\left\{\omega \in \Omega \mid X_{i}\left(\theta_{i}^{\prime \prime}\right)(\omega)>t\right\}\right]\right\} \mathrm{d} t .
\end{aligned}
$$

It follows from (47), (53), and (56) that

MTTF

$$
\begin{gathered}
=\frac{1}{2} \int_{0}^{1}\left\{\int_{0}^{+\infty}\left\{1-\prod_{i=1}^{n}\left[1-\operatorname{Pr}\left\{\omega \in \Omega \mid X_{i}\left(\theta_{i}^{\prime}\right)(\omega)>t\right\}\right]\right\} \mathrm{d} t\right. \\
+\int_{0}^{+\infty}\left\{1-\prod_{i=1}^{n}\left[1-\operatorname{Pr}\left\{\omega \in \Omega \mid X_{i}\left(\theta_{i}^{\prime \prime}\right)\right.\right.\right. \\
\times(\omega)>t\}]\} \mathrm{d} t\} \mathrm{d} \alpha \\
=\frac{1}{2} \int_{0}^{1}\left\{\int_{0}^{+\infty}\left\{1-\prod_{i=1}^{n}\left[1-\operatorname{Pr}_{\alpha}^{L}\left\{\omega \in \Omega \mid X_{i}\left(\theta_{i}\right)(\omega) \geq t\right\}\right]\right\} \mathrm{d} t\right. \\
+\int_{0}^{+\infty}\left\{1-\prod_{i=1}^{n}\left[1-\operatorname{Pr}_{\alpha}^{U}\left\{\omega \in \Omega \mid X_{i}\left(\theta_{i}\right)\right.\right.\right. \\
\times(\omega) \geq t\}]\} \mathrm{d} t\} \mathrm{d} \alpha \\
=\int_{0}^{+\infty} \frac{1}{2} \int_{0}^{1}\left\{1-\prod_{i=1}^{n}\left[1-\operatorname{Pr}_{\alpha}^{L}\left\{\omega \in \Omega \mid X_{i}\left(\theta_{i}\right)(\omega) \geq t\right\}\right]\right. \\
+1-\prod_{i=1}^{n}\left[1-\operatorname{Pr}_{\alpha}^{U}\left\{\omega \in \Omega \mid X_{i}\left(\theta_{i}\right)\right.\right. \\
\times \int_{0}^{+\infty} R(t) \mathrm{d} t .
\end{gathered}
$$

The theorem is proved.

Remark 30. If $X_{i}, i=1,2, \ldots, n$, degenerate to random variables, the result in Theorem 29 degenerates to the form

$$
\operatorname{MTTF}=\int_{0}^{+\infty} R(t) \mathrm{d} t
$$

in which $R(t)$ is the reliability of parallel system in stochastic case.

Remark 31. If $X_{i}, i=1,2, \ldots, n$, degenerate to fuzzy variables, the result in Theorem 29 degenerates to the form

$$
\operatorname{MTTF}=\int_{0}^{+\infty} R(t) \mathrm{d} t
$$

in which $R(t)$ is the reliability of parallel system in fuzzy case. 
Example 32. If the random fuzzy variable $X_{i}\left(\lambda_{i}\right) \sim$ $\mathscr{E} \mathscr{X} \mathscr{P}\left(\lambda_{i}\right)$, where $\lambda_{i}$ is a fuzzy variable on $\left(\Theta_{i}, \mathscr{P}\left(\Theta_{i}\right), \mathrm{Cr}_{i}\right)$, $i=1,2, \ldots, n$, then we can arrive at

$$
\begin{aligned}
& \operatorname{Pr}_{\alpha}^{L}\{\omega \in \Omega \mid X(\theta)(\omega) \geq t\}=1-\prod_{i=1}^{n}\left[1-e^{-\lambda_{i, \alpha}^{U} t}\right], \\
& \operatorname{Pr}_{\alpha}^{U}\{\omega \in \Omega \mid X(\theta)(\omega) \geq t\}=1-\prod_{i=1}^{n}\left[1-e^{-\lambda_{i, \alpha}^{L} t}\right] .
\end{aligned}
$$

By Theorems 26 and 29, we have

$$
\begin{gathered}
R(t)=1-\prod_{i=1}^{n}\left(1-e^{-E \lambda_{i} t}\right), \\
\operatorname{MTTF}=\int_{0}^{+\infty}\left\{1-\prod_{i=1}^{n}\left(1-e^{-E \lambda_{i} t}\right)\right\} \mathrm{d} t,
\end{gathered}
$$

in which " $E$ " is the expected value operator of fuzzy variable.

3.3. Reliability Analysis of Random Fuzzy Unrepairable SeriesParallel Systems. Consider a series-parallel system which is a series system of $m$ subsystems; each subsystem is composed of $n$ parallel components. Let $X_{i j}$ be the lifetime of component $j$ in $i$ th subsystem, which is a random fuzzy variable on the credibility space $\left(\Theta_{i j}, \mathscr{P}\left(\Theta_{i j}\right), \mathrm{Cr}_{i j}\right), i=1,2, \ldots, m$, $j=1,2, \ldots, n$. We assume the components are mutually independent. It is easy to know that the lifetime of the seriesparallel system is $X=\min _{1 \leq i \leq m}\left(\max _{1 \leq j \leq n} X_{i j}\right)$, which is a random fuzzy variable on the product credibility space $(\Theta, \mathscr{P}(\Theta)$, Cr $)$, where $\Theta=\Theta_{11} \times \Theta_{12} \times \cdots \times \Theta_{m n}$ and $\mathrm{Cr}=$ $\mathrm{Cr}_{11} \wedge \mathrm{Cr}_{12} \wedge \cdots \wedge \mathrm{Cr}_{m n}$.

Theorem 33. Let $X_{i j}, i=1,2, \ldots, m, j=1,2, \ldots, n$, be random fuzzy variables. Assume that the $\alpha$-pessimistic values and the $\alpha$-optimistic values of $E\left[X_{i j}\left(\theta_{i j}\right)\right], i=1,2, \ldots, m$, $j=1,2, \ldots, n$, are continuous at the point $\alpha, \alpha \in(0,1]$. The reliability of the series-parallel system is

$$
\begin{aligned}
& R(t) \\
& =E\left(\prod_{i=1}^{m}\left\{1-\prod_{j=1}^{n}\left[1-\operatorname{Pr}\left\{\omega \in \Omega \mid X_{i j}\left(\theta_{i j}\right)(\omega) \geq t\right\}\right]\right\}\right) .
\end{aligned}
$$

Remark 34. If $X_{i j}, i=1,2, \ldots, m, j=1,2, \ldots, n$, degenerate to random variables, the result in Theorem 33 degenerates to the form

$$
R(t)=\prod_{i=1}^{m}\left\{1-\prod_{j=1}^{n}\left[1-\operatorname{Pr}\left\{X_{i j} \geq t\right\}\right]\right\}
$$

which is consistent with the result in stochastic case (see Barlow and Proschan [1]).
Remark 35. If $X_{i j}, i=1,2, \ldots, m, j=1,2, \ldots, n$, degenerate to fuzzy variables, the result in Theorem 33 degenerates to the form

$$
R(t)=\operatorname{Cr}\left\{\min _{1 \leq i \leq m}\left(\max _{1 \leq j \leq n} X_{i j}\right) \geq t\right\},
$$

which is consistent with the result in fuzzy case (see Liu and Zhu [49]).

Theorem 36. Let $X_{i j}, i=1,2, \ldots, m, j=1,2, \ldots, n$, be random fuzzy variables. Assume that the $\alpha$-pessimistic values and the $\alpha$-optimistic values of $E\left[X_{i j}\left(\theta_{i j}\right)\right], i=1,2, \ldots, m$, $j=1,2, \ldots, n$, are continuous at the point $\alpha, \alpha \in(0,1]$. The MTTF of the random fuzzy series-parallel system is

$$
\text { MTTF }=\int_{0}^{+\infty} R(t) d t .
$$

Remark 37. If $X_{i j}, i=1,2, \ldots, m, j=1,2, \ldots, n$, degenerate to random variables, the result in Theorem 36 degenerates to the form

$$
\mathrm{MTTF}=\int_{0}^{+\infty} R(t) \mathrm{d} t
$$

in which $R(t)$ is the reliability of series-parallel system in stochastic case.

Remark 38. If $X_{i j}, i=1,2, \ldots, m, j=1,2, \ldots, n$, degenerate to fuzzy variables, the result in Theorem 36 degenerates to the form

$$
\operatorname{MTTF}=\int_{0}^{+\infty} R(t) \mathrm{d} t
$$

in which $R(t)$ is the reliability of series-parallel system in fuzzy case.

3.4. Reliability Analysis of Random Fuzzy Unrepairable Parallel-Series Systems. Consider a parallel-series system which is a parallel system of $m$ subsystems; each subsystem is composed of $n$ series components. Let $X_{i j}$ be the lifetime of component $j$ in $i$ th subsystem, which is a random fuzzy variable on the credibility space $\left(\Theta_{i j}, \mathscr{P}\left(\Theta_{i j}\right), \mathrm{Cr}_{i j}\right), i=$ $1,2, \ldots, m, j=1,2, \ldots, n$. We assume the components are mutually independent. It is easy to know that the lifetime of the parallel-series system is $X=\max _{1 \leq i \leq m}\left(\min _{1 \leq j \leq n} X_{i j}\right)$, which is a random fuzzy variable on the product credibility space $\left(\Theta, \mathscr{P}(\Theta)\right.$, Cr), where $\Theta=\Theta_{11} \times \Theta_{12} \times \cdots \times \Theta_{m n}$ and $\mathrm{Cr}=\mathrm{Cr}_{11} \wedge \mathrm{Cr}_{12} \wedge \cdots \wedge \mathrm{Cr}_{m n}$.

Theorem 39. Let $X_{i j}, i=1,2, \ldots, m, j=1,2, \ldots, n$, be random fuzzy variables. Assume that the $\alpha$-pessimistic values and the $\alpha$-optimistic values of $E\left[X_{i j}\left(\theta_{i j}\right)\right], i=1,2, \ldots, m$, $j=1,2, \ldots, n$, are continuous at the point $\alpha, \alpha \in(0,1]$. The reliability of the parallel-series system is

$R(t)$

$$
=1-E\left\{\prod_{i=1}^{m}\left[1-\prod_{j=1}^{n} \operatorname{Pr}\left\{\omega \in \Omega \mid X_{i j}\left(\theta_{i j}\right)(\omega) \geq t\right\}\right]\right\} .
$$


Remark 40. If $X_{i j}, i=1,2, \ldots, m, j=1,2, \ldots, n$, degenerate to random variables, the result in Theorem 39 degenerates to the form

$$
R(t)=1-\prod_{i=1}^{m}\left[1-\prod_{j=1}^{n} \operatorname{Pr}\left\{X_{i j} \geq t\right\}\right]
$$

which is consistent with the result in stochastic case (see Barlow and Proschan [1]).

Remark 41. If $X_{i j}, i=1,2, \ldots, m, j=1,2, \ldots, n$, degenerate to fuzzy variables, the result in Theorem 39 degenerates to the form

$$
R(t)=\operatorname{Cr}\left\{\max _{1 \leq i \leq m}\left(\min _{1 \leq j \leq n} X_{i j}\right) \geq t\right\}
$$

which is consistent with the result in fuzzy case (see Liu and Zhu [49]).

Theorem 42. Let $X_{i j}, i=1,2, \ldots, m, j=1,2, \ldots, n$, be random fuzzy variables. Assume that the $\alpha$-pessimistic values and the $\alpha$-optimistic values of $E\left[X_{i j}\left(\theta_{i j}\right)\right], i=1,2, \ldots, m$, $j=1,2, \ldots, n$, are continuous at the point $\alpha, \alpha \in(0,1]$. The MTTF of the random fuzzy parallel-series system is

$$
M T T F=\int_{0}^{+\infty} R(t) d t .
$$

Remark 43. If $X_{i j}, i=1,2, \ldots, m, j=1,2, \ldots, n$, degenerate to random variables, the result in Theorem 42 degenerates to the form

$$
\operatorname{MTTF}=\int_{0}^{+\infty} R(t) \mathrm{d} t
$$

in which $R(t)$ is the reliability of parallel-series system in stochastic case.

Remark 44. If $X_{i j}, i=1,2, \ldots, m, j=1,2, \ldots, n$, degenerate to fuzzy variables, the result in Theorem 42 degenerates to the form

$$
\operatorname{MTTF}=\int_{0}^{+\infty} R(t) \mathrm{d} t
$$

in which $R(t)$ is the reliability of parallel-series system in fuzzy case.

3.5. Reliability Analysis of Random fuzzy Unrepairable Cold Standby Systems. Consider a cold standby system composed of $n$ independent components, in which only one component is in operation. If the operating component fails, it will be replaced by another component. Suppose that the failed components are unrepairable and the components in standby do not deteriorate. The failure state of the system occurs only when there is no operative component left. We also assume that the switching mechanism is absolutely reliable. Let $X_{i}$ be the lifetime of component $i$, which is a random fuzzy variable on the credibility space $\left(\Theta_{i}, \mathscr{P}\left(\Theta_{i}\right), \mathrm{Cr}_{i}\right), i=1,2, \ldots, n$. The lifetime of the cold standby system can be expressed by the sum of the reliability of $n$ components; that is, $X=X_{1}+X_{2}+$ $\cdots+X_{n}$, which is a random fuzzy variable on the product credibility space $(\Theta, \mathscr{P}(\Theta), \mathrm{Cr})$, where $\Theta=\Theta_{1} \times \Theta_{2} \times \cdots \times \Theta_{n}$ and $\mathrm{Cr}=\mathrm{Cr}_{1} \wedge \mathrm{Cr}_{2} \wedge \cdots \wedge \mathrm{Cr}_{n}$.

Theorem 45. Let $X_{i}, i=1,2, \ldots, n$, be random fuzzy variables. Assume that the $\alpha$-pessimistic values and the $\alpha$ optimistic values of $E\left[X_{i}\left(\theta_{i}\right)\right], i=1,2, \ldots, n$, are continuous at the point $\alpha, \alpha \in(0,1]$. The reliability of random fuzzy cold standby system is

$$
\begin{aligned}
& R(t) \\
& \quad=E\left[\operatorname{Pr}\left\{\omega \in \Omega \mid X_{1}\left(\theta_{1}\right)(\omega)+\cdots+X_{n}\left(\theta_{n}\right)(\omega) \geq t\right\}\right] .
\end{aligned}
$$

Proof. By Definitions 13 and 17 and Proposition 7, we have

$$
\begin{aligned}
& R(t) \\
& =\operatorname{Ch}\{X \geq t\} \\
& =\int_{0}^{1} \operatorname{Cr}\{\theta \in \Theta \mid \operatorname{Pr}\{X(\theta) \geq t\} \geq p\} \mathrm{d} p \\
& =\frac{1}{2} \int_{0}^{1}\left(\operatorname{Pr}_{\alpha}^{L}\{\omega \in \Omega \mid X(\theta)(\omega) \geq t\}\right. \\
& \left.\quad+\operatorname{Pr}_{\alpha}^{U}\{\omega \in \Omega \mid X(\theta)(\omega) \geq t\}\right) \mathrm{d} \alpha \\
& =\frac{1}{2} \int_{0}^{1} \quad\left(\operatorname{Pr}_{\alpha}^{L}\left\{\omega \in \Omega \mid X_{1}\left(\theta_{1}\right)(\omega)+\cdots+X_{n}\left(\theta_{n}\right)(\omega) \geq t\right\}\right. \\
& \quad+\operatorname{Pr}_{\alpha}^{U}\left\{\omega \in \Omega \mid X_{1}\left(\theta_{1}\right)(\omega)\right. \\
& \left.\left.\quad+\cdots+X_{n}\left(\theta_{n}\right)(\omega) \geq t\right\}\right) \mathrm{d} \alpha \\
& =E\left[\operatorname{Pr}\left\{\omega \in \Omega \mid X_{1}\left(\theta_{1}\right)(\omega)+\cdots+X_{n}\left(\theta_{n}\right)(\omega) \geq t\right\}\right]
\end{aligned}
$$

which completes the proof.

Remark 46. If $X_{i}, i=1,2, \ldots, n$, degenerate to random variables, the result in Theorem 45 degenerates to the form

$$
R(t)=\operatorname{Pr}\left\{X_{1}+X_{2}+\cdots+X_{n} \geq t\right\},
$$

which is consistent with the result in stochastic case (see Barlow and Proschan [1]).

Remark 47. If $X_{i}, i=1,2, \ldots, n$, degenerate to fuzzy variables, the result in Theorem 45 degenerates to the form

$$
R(t)=\operatorname{Cr}\left\{X_{1}+X_{2}+\cdots+X_{n} \geq t\right\},
$$

which is consistent with the result in fuzzy case (see Liu and Zhu [49]).

Theorem 48. Let $X_{i}, i=1,2, \ldots, n$, be random fuzzy variables. Assume that the $\alpha$-pessimistic values and the $\alpha$ optimistic values of $E\left[X_{i}\left(\theta_{i}\right)\right], i=1,2, \ldots, n$, are continuous at 
the point $\alpha, \alpha \in(0,1]$. The MTTF of the cold standby system is

$$
\text { MTTF }=\int_{0}^{+\infty} R(t) d t .
$$

Proof. By Definition 18 and Proposition 7, we can see

$$
\begin{aligned}
\text { MTTF } & =\int_{0}^{+\infty} \operatorname{Cr}\{\theta \in \Theta \mid E[X(\theta)] \geq r\} \mathrm{d} r \\
& =\frac{1}{2} \int_{0}^{1}\left(E[X(\theta)]_{\alpha}^{L}+E[X(\theta)]_{\alpha}^{U}\right) \mathrm{d} \alpha .
\end{aligned}
$$

Let $A_{i}=\left\{\theta_{i} \in \Theta_{i} \mid \mu\left\{\theta_{i}\right\} \geq \alpha\right\}, i=1,2, \ldots, n$. Since the $\alpha$-pessimistic values and the $\alpha$-optimistic values of fuzzy variables $E\left[X_{i}\left(\theta_{i}\right)\right], \theta_{i} \in \Theta_{i}, i=1,2, \ldots, n$, are continuous at the point $\alpha, \alpha \in(0,1]$, then there exist maximum and minimum values; that is, at least there exist points $\theta_{i}^{\prime}, \theta_{i}^{\prime \prime} \in A_{i}$ such that

$$
E\left[X_{i}\left(\theta_{i}^{\prime}\right)\right]=E\left[X_{i}\left(\theta_{i}\right)\right]_{\alpha}^{L}, \quad E\left[X_{i}\left(\theta_{i}^{\prime \prime}\right)\right]=E\left[X_{i}\left(\theta_{i}\right)\right]_{\alpha}^{U} .
$$

For any $\theta_{i, \alpha} \in A_{i}, i=1,2, \ldots, n$, we have

$$
E\left[X_{i}\left(\theta_{i}^{\prime}\right)\right] \leq E\left[X_{i}\left(\theta_{i, \alpha}\right)\right] \leq E\left[X_{i}\left(\theta_{i}^{\prime \prime}\right)\right] .
$$

Hence, by Remark 16 we have

$$
X_{i}\left(\theta_{i}^{\prime}\right) \leq_{d} X_{i}\left(\theta_{i, \alpha}\right) \leq_{d} X_{i}\left(\theta_{i}^{\prime \prime}\right), \quad \theta_{i, \alpha} \in A_{i}, i=1,2, \ldots, n .
$$

Then

$$
\begin{aligned}
& X_{1}\left(\theta_{1}^{\prime}\right)+\cdots+X_{n}\left(\theta_{n}^{\prime}\right) \\
& \quad{ }_{d} X_{1}\left(\theta_{1, \alpha}\right)+\cdots+X_{n}\left(\theta_{n, \alpha}\right) \\
& \quad \leq{ }_{d} X_{1}\left(\theta_{1}^{\prime \prime}\right)+\cdots+X_{n}\left(\theta_{n}^{\prime \prime}\right) .
\end{aligned}
$$

It follows from Remark 16 that

$$
\begin{aligned}
E\left[X_{1}\right. & \left.\left(\theta_{1}^{\prime}\right)+\cdots+X_{n}\left(\theta_{n}^{\prime}\right)\right] \\
& \leq E\left[X_{1}\left(\theta_{1, \alpha}\right)+\cdots+X_{n}\left(\theta_{n, \alpha}\right)\right] \\
& \leq E\left[X_{1}\left(\theta_{1}^{\prime \prime}\right)+\cdots+X_{n}\left(\theta_{n}^{\prime \prime}\right)\right] .
\end{aligned}
$$

Since $\theta_{i, \alpha}$ are arbitrary points in $A_{i}, i=1,2, \ldots, n$, we have

$$
\begin{aligned}
& E[X(\theta)]_{\alpha}^{L} \\
& =E\left[X_{1}\left(\theta_{1}^{\prime}\right)+\cdots+X_{n}\left(\theta_{n}^{\prime}\right)\right] \\
& =\int_{0}^{+\infty} \operatorname{Pr}\left\{\omega \in \Omega \mid X_{1}\left(\theta_{1}^{\prime}\right)(\omega)+\cdots+X_{n}\left(\theta_{n}^{\prime}\right)(\omega) \geq t\right\} \mathrm{d} t, \\
& E[X(\theta)]_{\alpha}^{U} \\
& =E\left[X_{1}\left(\theta_{1}^{\prime \prime}\right)+\cdots+X_{n}\left(\theta_{n}^{\prime \prime}\right)\right] \\
& =\int_{0}^{+\infty} \operatorname{Pr}\left\{\omega \in \Omega \mid X_{1}\left(\theta_{1}^{\prime \prime}\right)(\omega)+\cdots+X_{n}\left(\theta_{n}^{\prime \prime}\right)(\omega) \geq t\right\} \mathrm{d} t .
\end{aligned}
$$

It follows from (79) and (85) that

MTTF

$$
\begin{aligned}
& =\frac{1}{2} \int_{0}^{1}\left\{\int _ { 0 } ^ { + \infty } \operatorname { P r } \left\{\omega \in \Omega \mid X_{1}\left(\theta_{1}^{\prime}\right)(\omega)\right.\right. \\
& \left.+\cdots+X_{n}\left(\theta_{n}^{\prime}\right)(\omega) \geq t\right\} \mathrm{d} t \\
& +\int_{0}^{+\infty} \operatorname{Pr}\left\{\omega \in \Omega \mid X_{1}\left(\theta_{1}^{\prime \prime}\right)(\omega)\right. \\
& +\cdots+X_{n}\left(\theta_{n}^{\prime \prime}\right) \\
& \times(\omega) \geq t\} \mathrm{d} t\} \mathrm{d} \alpha \\
& =\frac{1}{2} \int_{0}^{1} \int_{0}^{+\infty}\left\{\operatorname { P r } \left\{\omega \in \Omega \mid X_{1}\left(\theta_{1}^{\prime}\right)(\omega)\right.\right. \\
& \left.+\cdots+X_{n}\left(\theta_{n}^{\prime}\right)(\omega) \geq t\right\} \\
& +\operatorname{Pr}\left\{\omega \in \Omega \mid X_{1}\left(\theta_{1}^{\prime \prime}\right)(\omega)\right. \\
& \left.\left.+\cdots+X_{n}\left(\theta_{n}^{\prime \prime}\right)(\omega) \geq t\right\}\right\} \mathrm{d} t \mathrm{~d} \alpha .
\end{aligned}
$$

On the other hand, by (83) and Definition 15, we have

$$
\begin{aligned}
& \operatorname{Pr}\left\{\omega \in \Omega \mid X_{1}\left(\theta_{1}^{\prime}\right)+\cdots+X_{n}\left(\theta_{n}^{\prime}\right) \geq t\right\} \\
& \quad \leq \operatorname{Pr}\left\{\omega \in \Omega \mid X_{1}\left(\theta_{1, \alpha}\right)+\cdots+X_{n}\left(\theta_{n, \alpha}\right) \geq t\right\} \\
& \quad \leq \operatorname{Pr}\left\{\omega \in \Omega \mid X_{1}\left(\theta_{1}^{\prime \prime}\right)+\cdots+X_{n}\left(\theta_{n}^{\prime \prime}\right) \geq t\right\} .
\end{aligned}
$$

Since $\theta_{i, \alpha}$ are arbitrary points in $A_{i}, i=1,2, \ldots, n$, we have

$$
\begin{aligned}
\operatorname{Pr}_{\alpha}^{L}\{\omega & \left.\in \Omega \mid X_{1}\left(\theta_{1}\right)(\omega)+\cdots+X_{n}\left(\theta_{n}\right)(\omega) \geq t\right\} \\
& =\operatorname{Pr}\left\{\omega \in \Omega \mid X_{1}\left(\theta_{1}^{\prime}\right)+\cdots+X_{n}\left(\theta_{n}^{\prime}\right) \geq t\right\}, \\
\operatorname{Pr}_{\alpha}^{U}\{\omega & \left.\in \Omega \mid X_{1}\left(\theta_{1}\right)(\omega)+\cdots+X_{n}\left(\theta_{n}\right)(\omega) \geq t\right\} \\
& =\operatorname{Pr}\left\{\omega \in \Omega \mid X_{1}\left(\theta_{1}^{\prime \prime}\right)+\cdots+X_{n}\left(\theta_{n}^{\prime \prime}\right) \geq t\right\} .
\end{aligned}
$$

It follows from (86) and (88) that

MTTF

$$
\begin{aligned}
=\frac{1}{2} \int_{0}^{1} \int_{0}^{+\infty}\left\{\operatorname { P r } _ { \alpha } ^ { L } \left\{\omega \in \Omega \mid X_{1}\left(\theta_{1}\right)(\omega)\right.\right. \\
\left.+\cdots+X_{n}\left(\theta_{n}\right)(\omega) \geq t\right\} \\
+\operatorname{Pr}_{\alpha}^{U}\left\{\omega \in \Omega \mid X_{1}\left(\theta_{1}\right)(\omega)\right. \\
\left.\left.+\cdots+X_{n}\left(\theta_{n}\right)(\omega) \geq t\right\}\right\} \mathrm{d} t \mathrm{~d} \alpha
\end{aligned}
$$




$$
\begin{gathered}
=\int_{0}^{+\infty} \frac{1}{2} \int_{0}^{1}\left\{\operatorname { P r } _ { \alpha } ^ { L } \left\{\omega \in \Omega \mid X_{1}\left(\theta_{1}\right)(\omega)\right.\right. \\
\left.+\cdots+X_{n}\left(\theta_{n}\right)(\omega) \geq t\right\} \\
+\operatorname{Pr}_{\alpha}^{U}\left\{\omega \in \Omega \mid X_{1}\left(\theta_{1}\right)(\omega)\right. \\
\left.\left.+\cdots+X_{n}\left(\theta_{n}\right)(\omega) \geq t\right\}\right\} \mathrm{d} \alpha \mathrm{d} t \\
=\int_{0}^{+\infty} R(t) \mathrm{d} t
\end{gathered}
$$

The theorem is proved.

Remark 49. If $X_{i}, i=1,2, \ldots, n$, degenerate to random variables, the result in Theorem 48 degenerates to the form

$$
\operatorname{MTTF}=\int_{0}^{+\infty} R(t) \mathrm{d} t
$$

in which $R(t)$ is the reliability of cold standby system in stochastic case.

Remark 50. If $X_{i}, i=1,2, \ldots, n$, degenerate to fuzzy variables, the result in Theorem 48 degenerates to the form

$$
\operatorname{MTTF}=\int_{0}^{+\infty} R(t) \mathrm{d} t
$$

in which $R(t)$ is the reliability of cold standby system in fuzzy case.

\section{Numerical Examples}

In this section, we give the reliability analysis of a lighting lamp system and a hi-fi system with random fuzzy lifetimes.

Example 1. Consider a lighting lamp system composed by lamp $L_{1}$, lamp $L_{2}$, and lamp $L_{3}$; see Figure 1. The lifetimes of lamp $L_{1}$, lamp $L_{2}$, and lamp $L_{3}$ are denoted by $X_{1}, X_{2}$, and $X_{3}$, respectively. The lifetime of the lighting lamp system is denoted by $X$. We also assume $X_{1}, X_{2}$, and $X_{3}$ to be random fuzzy variables on $(\Theta, \mathscr{P}(\Theta), \mathrm{Cr})$ and $X_{i}\left(\lambda_{i}\right) \sim \mathscr{E} \mathscr{X} \mathscr{P}\left(\lambda_{i}\right), i=$ $1,2,3$, where $\lambda_{1}=(1,2,3), \lambda_{2}=(0,1,2)$, and $\lambda_{3}=(0,1,2)$. Then we can arrive at

$$
\begin{array}{cc}
\lambda_{1, \alpha}^{L}=1+\alpha, & \lambda_{1, \alpha}^{U}=3-\alpha, \\
\lambda_{2, \alpha}^{L}=\alpha, & \lambda_{2, \alpha}^{U}=2-\alpha, \\
\lambda_{3, \alpha}^{L}=\alpha, & \lambda_{3, \alpha}^{U}=2-\alpha .
\end{array}
$$

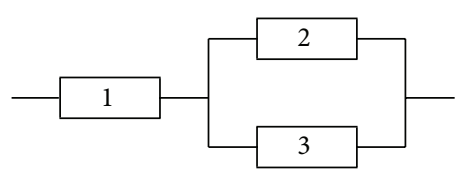

FIGURE 1: A lighting lamp system.

Then we have

$$
\begin{aligned}
\operatorname{Pr}_{\alpha}^{L}\{X \geq t\} \\
=\operatorname{Pr}_{\alpha}^{L}\left\{X_{1} \geq t\right\} \\
\quad \times\left\{1-\left(1-\operatorname{Pr}_{\alpha}^{L}\left\{X_{2} \geq t\right\}\right)\left(1-\operatorname{Pr}_{\alpha}^{L}\left\{X_{3} \geq t\right\}\right)\right\} \\
=e^{-(3-\alpha) t}\left\{1-\left(1-e^{-(2-\alpha) t}\right)\left(1-e^{-(2-\alpha) t}\right)\right\} \\
=2 e^{-(5-2 \alpha) t}-e^{-(7-3 \alpha) t}, \\
\operatorname{Pr}_{\alpha}^{U}\{X \geq t\} \\
=\operatorname{Pr}_{\alpha}^{U}\left\{X_{1} \geq t\right\} \\
\quad \times\left\{1-\left(1-\operatorname{Pr}_{\alpha}^{U}\left\{X_{2} \geq t\right\}\right)\left(1-\operatorname{Pr}_{\alpha}^{U}\left\{X_{3} \geq t\right\}\right)\right\} \\
=e^{-(1+\alpha) t}\left\{1-\left(1-e^{-\alpha t}\right)\left(1-e^{-\alpha t}\right)\right\} \\
=2 e^{-(1+2 \alpha) t}-e^{-(1+3 \alpha) t} .
\end{aligned}
$$

So the reliability of the lighting lamp system is

$$
\begin{aligned}
& R(t) \\
& =\frac{1}{2} \int_{0}^{1}\left[2 e^{-(5-2 \alpha) t}-e^{-(7-3 \alpha) t}+2 e^{-(1+2 \alpha) t}-e^{-(1+3 \alpha) t}\right] \mathrm{d} \alpha \\
& =\frac{1}{6 t}\left(2 e^{-t}-3 e^{-5 t}+e^{-7 t}\right) .
\end{aligned}
$$

The MTTF of the lighting lamp system is

$$
\operatorname{MTTF}=\int_{0}^{+\infty} \frac{1}{6 t}\left(2 e^{-t}-3 e^{-5 t}+e^{-7 t}\right) \mathrm{d} t \approx 0.4804
$$

Example 2. A more elaborate example is a stereo hi-fi system with the following components: (1) FM tuner; (2) record changer; (3) amplifier; (4) speaker A; and (5) speaker B. We consider the system functioning if we can obtain music (monaural or stereo) through FM or records. The system structure is illustrated in Figure 2. Let $X_{i}$ be the random fuzzy lifetime of component $i, i=1,2, \ldots, 5$. The lifetime of the hifi system is denoted by $X$. We assume $X_{i}\left(\lambda_{i}\right) \sim \mathscr{E} \mathscr{X} \mathscr{P}\left(\lambda_{i}\right)$, $i=1,2, \ldots, 5$, where $\lambda_{1}=(1,2,3), \lambda_{2}=(1.5,2.5,3.5)$, $\lambda_{3}=(0,1,2), \lambda_{4}=(1,2,3)$, and $\lambda_{5}=(0.5,1.5,2.5)$. 


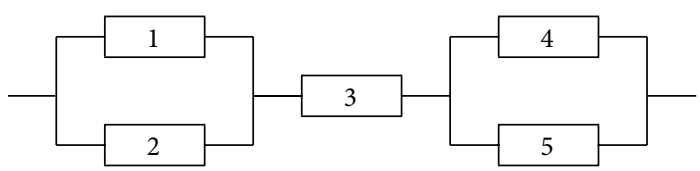

Figure 2: A hi-fi system.

We first compute

$$
\begin{array}{cl}
\lambda_{1, \alpha}^{L}=1+\alpha, & \lambda_{1, \alpha}^{U}=3-\alpha, \\
\lambda_{2, \alpha}^{L}=1.5+\alpha, & \lambda_{2, \alpha}^{U}=3.5-\alpha, \\
\lambda_{3, \alpha}^{L}=\alpha, & \lambda_{3, \alpha}^{U}=2-\alpha, \\
\lambda_{4, \alpha}^{L}=1+\alpha, & \lambda_{4, \alpha}^{U}=3-\alpha, \\
\lambda_{5, \alpha}^{L}=0.5+\alpha, & \lambda_{5, \alpha}^{U}=2.5-\alpha .
\end{array}
$$

Then we can arrive at

$$
\begin{aligned}
& \operatorname{Pr}_{\alpha}^{L}\{X \geq t\} \\
& =\left\{1-\left(1-\operatorname{Pr}_{\alpha}^{L}\left\{X_{1} \geq t\right\}\right)\left(1-\operatorname{Pr}_{\alpha}^{L}\left\{X_{2} \geq t\right\}\right)\right\} \\
& \times \operatorname{Pr}_{\alpha}^{L}\left\{X_{3} \geq t\right\} \\
& \cdot\left\{1-\left(1-\operatorname{Pr}_{\alpha}^{L}\left\{X_{4} \geq t\right\}\right)\left(1-\operatorname{Pr}_{\alpha}^{L}\left\{X_{5} \geq t\right\}\right)\right\} \\
& =\left\{1-\left[1-e^{-(3-\alpha) t}\right]\left[1-e^{-(3.5-\alpha) t}\right]\right\} e^{-(2-\alpha) t} \\
& \times\left\{1-\left[1-e^{-(3-\alpha) t}\right]\left[1-e^{-(2.5-\alpha) t}\right]\right\} \\
& =e^{-(2-\alpha) t}\left[e^{-(3.5-\alpha) t}+e^{-(3-\alpha) t}-e^{-(6.5-2 \alpha) t}\right] \\
& \times\left[e^{-(3-\alpha) t}+e^{-(2.5-\alpha) t}-e^{-(5.5-2 \alpha) t}\right] \text {, } \\
& \operatorname{Pr}_{\alpha}^{U}\{X \geq t\} \\
& =\left\{1-\left(1-\operatorname{Pr}_{\alpha}^{U}\left\{X_{1} \geq t\right\}\right)\left(1-\operatorname{Pr}_{\alpha}^{U}\left\{X_{2} \geq t\right\}\right)\right\} \\
& \times \operatorname{Pr}_{\alpha}^{U}\left\{X_{3} \geq t\right\} \\
& \cdot\left\{1-\left(1-\operatorname{Pr}_{\alpha}^{U}\left\{X_{4} \geq t\right\}\right)\left(1-\operatorname{Pr}_{\alpha}^{U}\left\{X_{5} \geq t\right\}\right)\right\} \\
& =\left\{1-\left[1-e^{-(1+\alpha) t}\right]\left[1-e^{-(1.5+\alpha) t}\right]\right\} e^{-\alpha t} \\
& \times\left\{1-\left[1-e^{-(1+\alpha) t}\right]\left[1-e^{-(0.5+\alpha) t}\right]\right\} \\
& =e^{-\alpha t}\left[e^{-(1+\alpha) t}+e^{-(1.5+\alpha) t}-e^{-(2.5+2 \alpha) t}\right] \\
& \times\left[e^{-(1+\alpha) t}+e^{-(0.5+\alpha) t}-e^{-(1.5+2 \alpha) t}\right] .
\end{aligned}
$$

The reliability of the hi-fi system is

$$
\begin{aligned}
& R(t) \\
& =\frac{1}{2} \int_{0}^{1}\left\{e^{-(2-\alpha) t}\left[e^{-(3.5-\alpha) t}+e^{-(3-\alpha) t}-e^{-(6.5-2 \alpha) t}\right]\right. \\
& \times\left[e^{-(3-\alpha) t}+e^{-(2.5-\alpha) t}-e^{-(5.5-2 \alpha) t}\right] \\
& +e^{-\alpha t}\left[e^{-(1+\alpha) t}+e^{-(1.5+\alpha) t}-e^{-(2.5+2 \alpha) t}\right] \\
& \left.\times\left[e^{-(1+\alpha) t}+e^{-(0.5+\alpha) t}-e^{-(1.5+2 \alpha) t}\right]\right\} \mathrm{d} \alpha \\
& =\frac{1}{6 t}\left(2 e^{-2 t}+e^{-1.5 t}+e^{-2.5 t}-e^{-8.5 t}-2 e^{-8 t}-e^{-7.5 t}\right) \\
& +\frac{1}{10 t}\left(e^{-4 t}-e^{-14 t}\right) \\
& +\frac{1}{8 t}\left(2 e^{-10 t}-2 e^{-6 t}+e^{-10.5 t}+e^{-11.5 t}\right. \\
& \left.-e^{-2.5 t}+2 e^{-7 t}-2 e^{-3 t}-e^{-3.5 t}\right) \text {. }
\end{aligned}
$$

The MTTF of the hi-fi system is

\section{MTTF}

$$
\begin{gathered}
\int_{0}^{+\infty}\left[\frac{1}{6 t}\left(2 e^{-2 t}+e^{-1.5 t}+e^{-2.5 t}-e^{-8.5 t}-2 e^{-8 t}-e^{-7.5 t}\right)\right. \\
+\frac{1}{10 t}\left(e^{-4 t}-e^{-14 t}\right) \\
+\frac{1}{8 t}\left(2 e^{-10 t}-2 e^{-6 t}+e^{-10.5 t}+e^{-11.5 t}\right. \\
\left.\left.-e^{-2.5 t}+2 e^{-7 t}-2 e^{-3 t}-e^{-3.5 t}\right)\right] \mathrm{d} t
\end{gathered}
$$

$\approx 0.3921$.

\section{Conclusion}

In this paper, the random fuzzy theory provides a mathematical foundation for the reliability theory, which makes it possible to solve more complex unrepairable systems with fuzziness and randomness. Based on that, we establish five basic mathematical models of random fuzzy unrepairable systems, including series systems, parallel systems, seriesparallel systems, parallel-series systems, and cold standby systems with absolutely reliable conversion switches. Furthermore, the expressions of reliability and MTTF are given for the above five random fuzzy unrepairable systems, respectively. When the random fuzzy lifetimes degenerate to random lifetimes or fuzzy lifetimes, the results we arrived at are also suitable. In future research, continuous attention might be paid to random fuzzy systems, and we should give reliability analysis or discuss the maintenance policy of repairable systems. 


\section{Conflict of Interests}

The authors declare that there is no conflict of interests regarding the publication of this paper.

\section{Acknowledgment}

This work was supported by the National Natural Science Foundation of China, Grant nos. 11301382, 61070021, and 71171088. The research is also supported by NCET-12-0081 and the Fundamental Research Funds for the Central Universities, HUST: CXY12M013.

\section{References}

[1] R. E. Barlow and F. Proschan, Statistical Theory of Reliability and Life Testing: Probability Models, Holt, Rinehart and Winston, New York, NY, USA, 1975.

[2] B. S. Dhillon and C. Singh, Engineering Reliability: New Techniques and Applications, John Wiley \& Sons, New York, NY, USA, 1981.

[3] B. Epstein and M. Sobel, "Some theorems relevant to life testing from an exponential distribution," Annals of Mathematical Statistics, vol. 25, pp. 373-381, 1954.

[4] B. V. Gnedenko, Yu. K. Belyayev, and A. D. Solovyev, Mathematical Methods of Reliability Theory, Academic Press, New York, NY, USA, 1969.

[5] A. Kaufmann, Introduction to the Theory of Fuzzy Subsets. Volume I: Fundamental Theoretical Elements, Academic Press, New York, NY, USA, 1975.

[6] K. C. Kapur and L. R. Lamberson, "Optimum test design strategies," Microelectronics Reliability, vol. 20, no. 1-2, pp. 7581, 1980.

[7] R. Natarajan, "A reliability problem with spares and multiple repair facilities," Operations Research, vol. 16, pp. 1041-1057, 1968.

[8] S. M. Ross, Applied Probability Models with Optimization Applications, Holden-Day, San Francisco, Calif, USA, 1970.

[9] V. K. Sharma, M. Agarwal, and K. Sen, "Reliability evaluation and optimal design in heterogeneous multi-state series-parallel systems," Information Sciences, vol. 181, no. 2, pp. 362-378, 2011.

[10] Y.-K. Lin and C.-T. Yeh, "Computer network reliability optimization under double-resource assignments subject to a transmission budget," Information Sciences, vol. 181, no. 3, pp. 582599, 2011.

[11] Z. Tian, G. Levitin, and M. J. Zuo, "A joint reliabilityredundancy optimization approach for multi-state seriesparallel systems," Reliability Engineering and System Safety, vol. 94, no. 10, pp. 1568-1576, 2009.

[12] D. Marquez, M. Neil, and N. Fenton, "Improved reliability modeling using Bayesian networks and dynamic discretization," Reliability Engineering and System Safety, vol. 95, no. 4, pp. 412425, 2010.

[13] C.-J. Hsu, T. C. E. Cheng, and D.-L. Yang, "Unrelated parallelmachine scheduling with rate-modifying activities to minimize the total completion time," Information Sciences, vol. 181, no. 20, pp. 4799-4803, 2011.

[14] S. G. Chowdhury and K. B. Misra, "Evaluation of fuzzy reliability of a non-series parallel network," Microelectronics Reliability, vol. 32, no. 1-2, pp. 1-4, 1992.
[15] K. Y. Cai, C. Y. Wen, and M. L. Zhang, "Fuzzy variables as a basis for a theory of fuzzy reliability in the possibility context," Fuzzy Sets and Systems, vol. 42, no. 2, pp. 145-172, 1991.

[16] C. Kai-Yuan, "Posbist reliability behavior of fault-tolerant systems," Microelectronics Reliability, vol. 35, no. 1, pp. 49-56, 1995.

[17] K. Y. Cai, C. Y. Wen, and M. L. Zhang, "Posbist reliability behavior of typical systems with two types of failure," Fuzzy Sets and Systems, vol. 43, no. 1, pp. 17-32, 1991.

[18] K.-Y. Cai, C.-Y. Wen, and M.-L. Zhang, "Fuzzy states as a basis for a theory of fuzzy reliability," Microelectronics Reliability, vol. 33, no. 15, pp. 2253-2263, 1993.

[19] L. V. Utkin, "Knowledge based fuzzy reliability assessment," Microelectronics Reliability, vol. 34, no. 5, pp. 863-874, 1994.

[20] L. V. Utkin, "Fuzzy reliability of repairable systems in the possibility context," Microelectronics Reliability, vol. 34, no. 12, pp. 1865-1876, 1994.

[21] L. V. Utkin and S. V. Gurov, "A general formal approach for fuzzy reliability analysis in the possibility context," Fuzzy Sets and Systems, vol. 83, no. 2, pp. 203-213, 1996.

[22] B. Praba, R. Sujatha, and S. Srikrishna, "Posfust reliability of a unified fuzzy Markov model," International Journal of Reliability and Safety, vol. 5, no. 1, pp. 83-94, 2011.

[23] G. de Cooman, "On modeling possibilistic uncertainty in twostate reliability theory," Fuzzy Sets and Systems, vol. 83, no. 2, pp. 215-238, 1996.

[24] H.-Z. Huang, "Reliability analysis method in the presence of fuzziness attached to operating time," Microelectronics Reliability, vol. 35, no. 12, pp. 1483-1487, 1995.

[25] H.-Z. Huang, M. J. Zuo, and Z.-Q. Sun, "Bayesian reliability analysis for fuzzy lifetime data," Fuzzy Sets and Systems, vol. 157, no. 12, pp. 1674-1686, 2006.

[26] Y. Liu, H. Huang, and G. Levitin, "Reliability and performance assessment for fuzzy multi-state elements," Journal of Risk and Reliability, vol. 222, pp. 675-686, 2008.

[27] Y. Ding and A. Lisnianski, "Fuzzy universal generating functions for multi-state system reliability assessment," Fuzzy Sets and Systems, vol. 159, no. 3, pp. 307-324, 2008.

[28] Q. Jiang and C.-H. Chen, "A numerical algorithm of fuzzy reliability," Reliability Engineering and System Safety, vol. 80, no. 3, pp. 299-307, 2003.

[29] J. Zhang, R. Zhao, and W. Tang, "Fuzzy age-dependent replacement policy and SPSA algorithm based-on fuzzy simulation," Information Sciences, vol. 178, no. 2, pp. 573-583, 2008.

[30] O. Linda and M. Manic, "Interval type-2 fuzzy voter design for fault tolerant systems," Information Sciences, vol. 181, no. 14, pp. 2933-2950, 2011.

[31] S. Wang and J. Watada, "Fuzzy random renewal reward process and its applications," Information Sciences, vol. 179, no. 23, pp. 4057-4069, 2009.

[32] S. Wang and J. Watada, "Modelling redundancy allocation for a fuzzy random parallel-series system," Journal of Computational and Applied Mathematics, vol. 232, no. 2, pp. 539-557, 2009.

[33] P. R. Adduri and R. C. Penmetsa, "System reliability analysis for mixed uncertain variables," Structural Safety, vol. 31, no. 5, pp. 375-382, 2009.

[34] L. V. Utkin and F. P. A. Coolen, "Imprecise reliability: an introductory overview," Studies in Computational Intelligence, vol. 40, pp. 261-306, 2007.

[35] L. V. Utkin, S. V. Gurov, and I. B. Shubinsky, "Reliability of systems by mixture forms of uncertainty," Microelectronics Reliability, vol. 37, no. 5, pp. 779-783, 1997. 
[36] L.-L. Li, F.-F. Zhu, C.-W. Yang, and Z.-G. Li, "Research on the credibility of fuzzy reliability," Applied Mechanics and Materials, vol. 48-49, pp. 984-988, 2011.

[37] Y. Liu, Z. Qiao, and G. Wang, "Fuzzy random reliability of structures based on fuzzy random variables," Fuzzy Sets and Systems, vol. 86, no. 3, pp. 345-355, 1997.

[38] B. Liu, Uncertainty Theory: Toward Axiomatic Foundations, Lecture Note, Tsinghua University, 2003.

[39] R. Zhao, W. Tang, and H. Yun, "Random fuzzy renewal process," European Journal of Operational Research, vol. 169, no. 1, pp. 189-201, 2006.

[40] R. Zhao and B. Liu, "Redundancy optimization problems with uncertainty of combining randomness and fuzziness," European Journal of Operational Research, vol. 157, no. 3, pp. 716-735, 2004.

[41] Y. Liu, X. Li, and G. Yang, "Reliability analysis of random fuzzy repairable series system," Advances in Intelligent and Soft Computing, vol. 78, pp. 281-296, 2010.

[42] Y. Liu, W. Tang, and X. Li, "Random fuzzy shock models and bivariate random fuzzy exponential distribution," Applied Mathematical Modelling, vol. 35, no. 5, pp. 2408-2418, 2011.

[43] B. Liu and Y.-K. Liu, "Expected value of fuzzy variable and fuzzy expected value models," IEEE Transactions on Fuzzy Systems, vol. 10, no. 4, pp. 445-450, 2002.

[44] B. Liu, "A survey of credibility theory," Fuzzy Optimization and Decision Making, vol. 5, no. 4, pp. 387-408, 2006.

[45] B. Liu, Theory and Practice of Uncertainty Programming, Physica, Heidelberg, Germany, 2002.

[46] Y.-K. Liu and B. Liu, "Expected value operator of random fuzzy variable and random fuzzy expected value models," International Journal of Uncertainty, Fuzziness and KnowledgeBased Systems, vol. 11, no. 2, pp. 195-215, 2003.

[47] Y.-K. Liu and B. Liu, "Random fuzzy programming with chance measures defined by fuzzy integrals," Mathematical and Computer Modelling, vol. 36, no. 4-5, pp. 509-524, 2002.

[48] S. M. Ross, Stochastic Processes, Wiley Series in Probability and Statistics: Probability and Statistics, John Wiley \& Sons, New York, NY, USA, 2nd edition, 1996.

[49] Y. Liu and H. Zhu, "Reliability analysis of fuzzy unrepairable systems," Information, vol. 15, no. 10, pp. 3935-3944, 2012. 


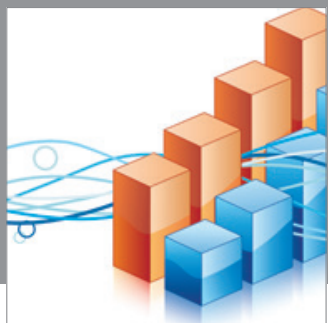

Advances in

Operations Research

mansans

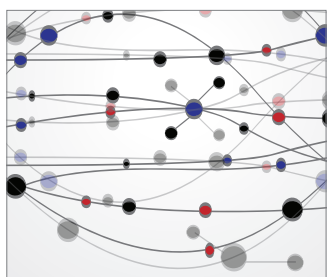

The Scientific World Journal
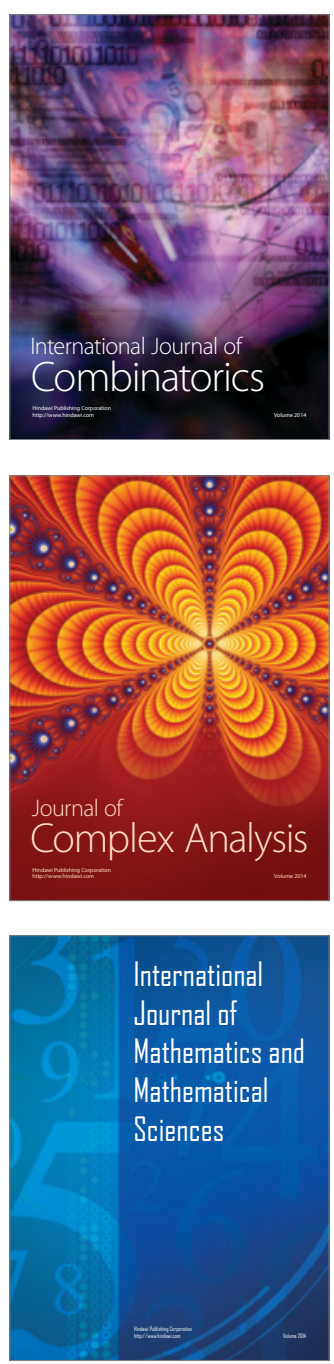
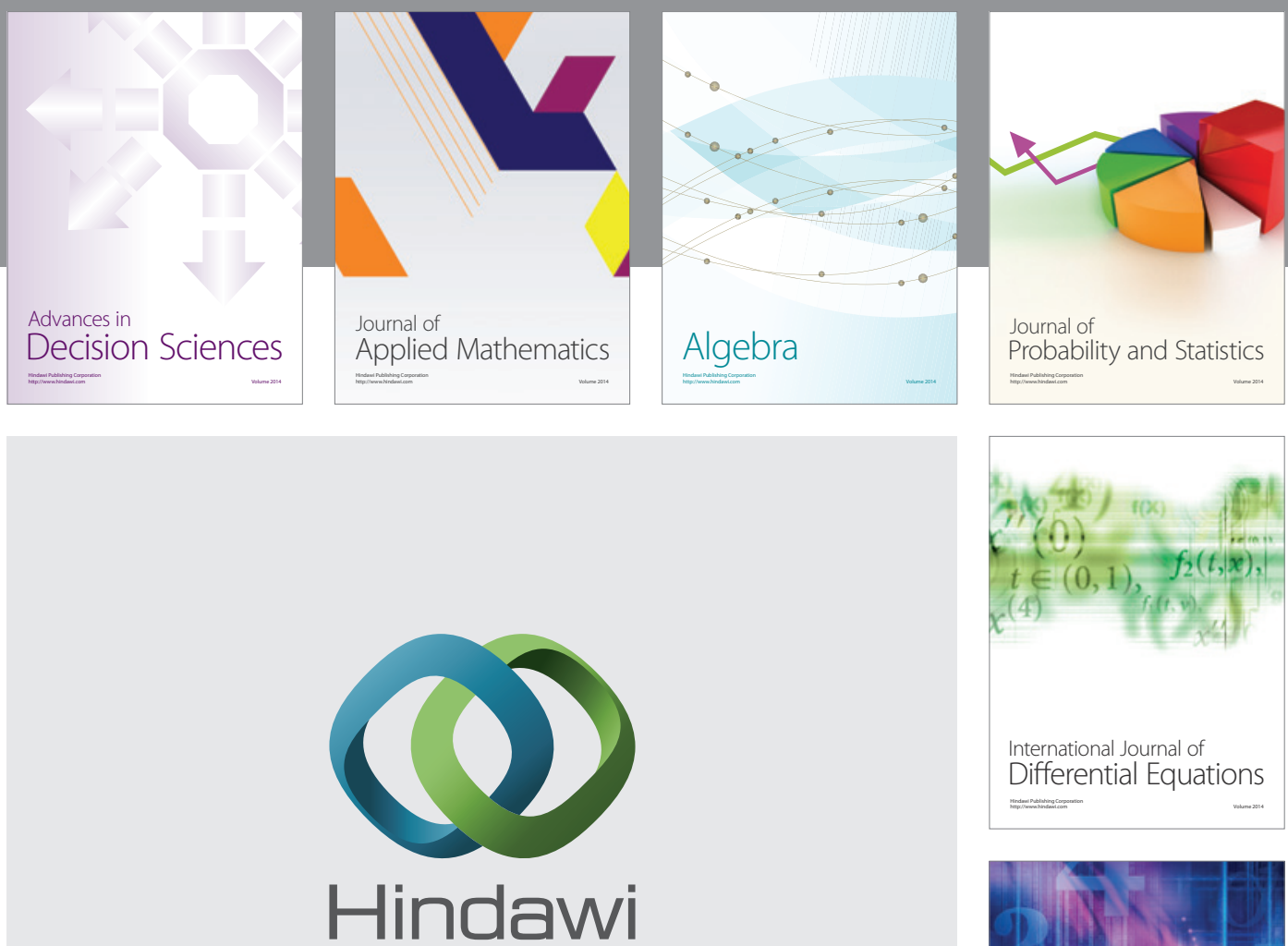

Submit your manuscripts at http://www.hindawi.com
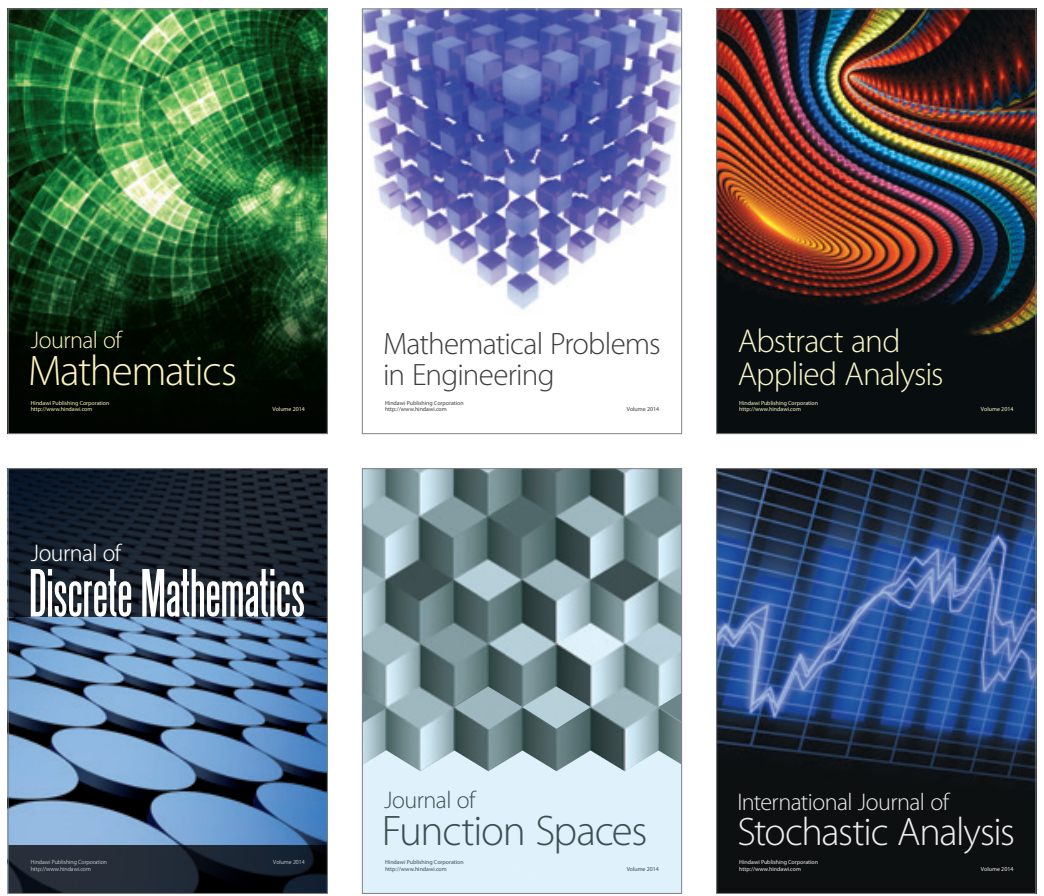

Journal of

Function Spaces

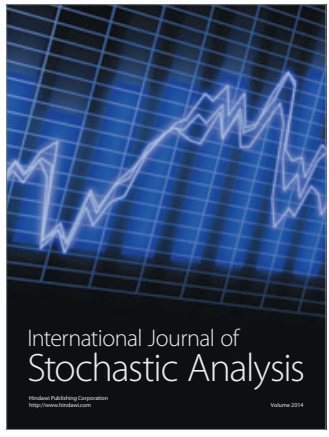

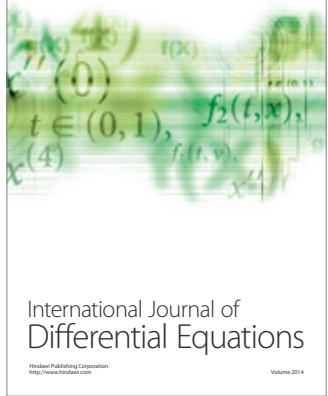
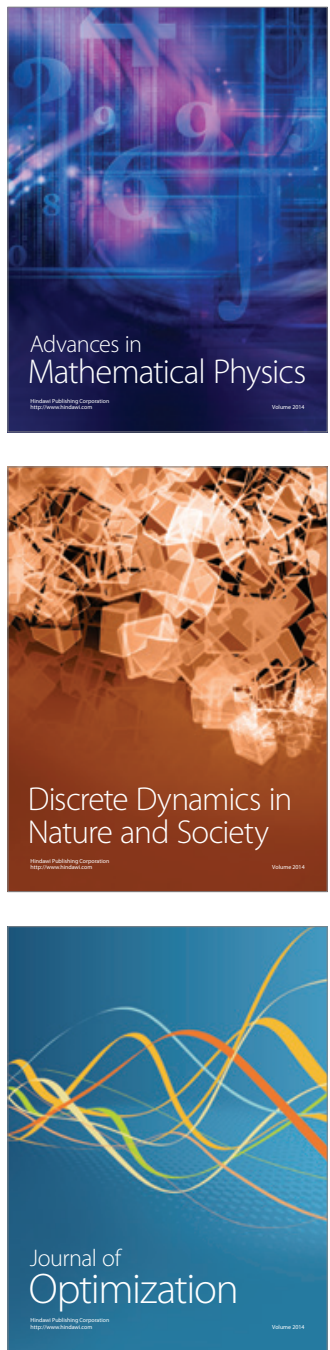